\title{
Global Existence and Decay of Solutions for Coupled Nondegenerate Kirchhoff System with a Time Varying Delay Term
}

\author{
Nadia Mezouar (D), ${ }^{1}$ Salah Mahmoud Boulaaras $\mathbb{D D}^{2,3}$ Sultan Alodhaibi ${ }^{2},^{2}$ \\ and Salem Alkhalaf ${ }^{4}$ \\ ${ }^{1}$ Department of Commerce, Faculty of Economic, Mascara University, 29000 Mascara, Algeria \\ ${ }^{2}$ Department of Mathematics, College of Sciences and Arts, Qassim University, Al-Rass, Saudi Arabia \\ ${ }^{3}$ Laboratory of Fundamental and Applied Mathematics of Oran (LMFAO), University of Oran 1, Ahmed Benbella, Algeria \\ ${ }^{4}$ Department of Computer, College of Sciences and Arts, Qassim University, Al-Rass, Saudi Arabia
}

Correspondence should be addressed to Salah Mahmoud Boulaaras; s.boularas@qu.edu.sa

Received 14 January 2020; Accepted 28 March 2020; Published 9 May 2020

Guest Editor: Shao-Bo He

Copyright (c) 2020 Nadia Mezouar et al. This is an open access article distributed under the Creative Commons Attribution License, which permits unrestricted use, distribution, and reproduction in any medium, provided the original work is properly cited.

\begin{abstract}
This paper deals with the global existence of solutions in a bounded domain for nonlinear viscoelastic Kirchhoff system with a time varying delay by using the energy and Faedo-Galerkin method with respect to the delay term weight condition in the feedback and the delay speed. Furthermore, by using some convex functions properties, we prove a uniform stability estimate.
\end{abstract}

\section{Introduction}

1.1. Model. Consider the following viscoelastic Kirchhoff system:

$$
\begin{cases}\left|u_{t}\right|^{l} u_{t t}+\alpha v-M\left(\|\nabla u\|^{2}\right) \Delta u-\Delta u_{t t}+\int_{0}^{t} h_{1}(t-s) \Delta u(s) \mathrm{d} s-\mu_{1} \Delta u_{t}(x, t-\tau(t))=0, & \text { in } \Omega \times] 0,+\infty[, \\ \left|v_{t}\right|^{l} v_{t t}+\alpha u-M\left(\|\nabla v\|^{2}\right) \Delta v-\Delta v_{t t}+\int_{0}^{t} h_{2}(t-s) \Delta v(s) \mathrm{d} s-\mu_{2} \Delta v_{t}(x, t-\tau(t))=0, & \text { in } \Omega \times] 0,+\infty[, \\ u(x, t)=v(x, t)=0, & \text { on } \partial \Omega \times] 0,+\infty[, \\ (u(x, 0), v(x, 0))=\left(u_{0}(x), v_{0}(x)\right),\left(u_{t}(x, 0), v_{t}(x, 0)\right)=\left(u_{1}(x), v_{1}(x)\right), & \text { in } \Omega, \\ \left(u_{t}(x, t-\tau(0)), v_{t}(x, t-((0)))=\left(f_{0}(x, t-\tau(0)), g_{0}(x, t-\tau(0))\right),\right. & \text { in } \Omega \times] 0, \tau(0)[,\end{cases}
$$

where $\Omega$ is a bounded domain in $\mathrm{IR}^{n}, n \in \mathrm{IN}^{*}$, with a smooth boundary $\partial \Omega, l>0, \alpha, \mu_{1}$ and $\mu_{2}$ are positive real numbers, $h_{1}$ and $h_{2}$ are positive functions which decay exponentially, $\tau(t)>0$ is a time varying delay, and the initial data $\left(u_{0}, v_{0}, u_{1}, v_{1}, f_{0}, g_{0}\right)$ are in a suitable function space.
$M(r)=a+b r^{\gamma}$ is a $C^{1}$-function for $r \geq 0$, with $a, b>0$, and $\gamma \geq 1$.

Time delay is often present in applications and practical problems. In last few years, the control of PDEs with time delay effects has become an active area of research (see, for 
example, [1-4] and the references therein). In [5], the authors showed that a small delay in a boundary control could turn a well-behaved hyperbolic system into a wild one and therefore delay becomes a source of instability. However, sometimes it can also improve the performance of the system.

By using the Faedo-Galerkin method, Wu in [6] proved the result of local existence and established the decay result by suitable Lyapunov functionals according to appropriate conditions on $\mu_{1}, \mu_{2}$ and on the kernel $h$.

Daewook [7] studied the following viscoelastic Kirchhoff equation with nonlinear source term and varying time delay:

$$
\begin{aligned}
u_{t t}- & M\left(x, t,\|\nabla u\|^{2}\right) \Delta u+\int_{0}^{t} h(t-s) \operatorname{div}(a(x) \nabla u(s)) \mathrm{d} s \\
+ & |u|^{m} u+\mu_{1} u_{t}(x, t)+\mu_{2} u_{t}(x, t-\tau(t))=0, \\
& \text { in } \Omega \times] 0,+\infty[,
\end{aligned}
$$

which is a description of axially moving viscoelastic materials. According to the smallness condition taking into account of Kirchhoff coefficient and the relaxation function and by summing $0 \leq m \leq(2 /(n-2))$ if $n>2$ or $0 \leq m$ if $n \leq 2$, he got the uniform decay rate of the Kirchhoff type energy.

Very recently, in [1], we have proved the global existence and energy decay of solutions of the following viscoelastic nondegenerate Kirchhoff equation:

$$
\begin{cases}\left|u_{t}\right|^{l} u_{t t}-M\left(\|\nabla u\|^{2}\right) \Delta u-\Delta u_{t t}+\int_{0}^{t} h(t-s) \Delta u(s) \mathrm{d} s+\mu_{1} g_{1}\left(u_{t}(x, t)\right)+\mu_{2} g_{2}\left(u_{t}(x, t-\tau(t))\right)=0, & \text { in } \Omega \times] 0,+\infty[, \\ u(x, t)=0, & \text { on }] \partial \Omega \times[0,+\infty, \\ u(x, 0)=u_{0}(x), u_{t}(x, 0)=u_{1}(x), & \text { in } \Omega, \\ u_{t}(x, t-\tau(0))=f_{0}(x, t-\tau(0)), & \text { in } \Omega \times] 0, \tau(0)[,\end{cases}
$$

with respect to some proposed assumptions. Under assumption setting on $g_{1}, g_{2}, \sigma$, and $\tau$, the authors have obtained the global existence of solution and the decay rate of energy.

Recently, Mezouar and Boulaaras [1] have studied viscoelastic nondegenerate Kirchhoff equation with varying delay term in the internal feedback.

In the present paper, we extent our recent published paper in [1] for a coupled system (3). The famous technique using the presence of delay in PDE's problem is to set a new variable defined by velocity depending on delay, which will give us new problem equivalent to our studied problem but the last one is a coupled system without delay. After this, we can prove the existence of global solutions in suitable Sobolev spaces by combining the energy method with the Faedo-Galerkin procedure, and under a choice of a suitable Lyapunov functional, we establish an exponential decay result.

The outline of the paper is as follows. In Section 2, some hypotheses related to problem are given and we state our main result. Then, in Section 3, the global existence of weak solutions is proven. Finally, in Section 4, we give the uniform energy decay.
1.2. Preliminaries and Assumptions. We denote by (...) the inner product in $L^{2}(\Omega)$.

Now, we introduce, as in [8], the new variables

$$
\begin{array}{ll}
z_{1}(x, \rho, t)=u_{t}(x, t-\rho \tau(t)), & x \in \Omega, \rho \in(0,1), t>0, \\
z_{2}(x, \rho, t)=v_{t}(x, t-\rho \tau(t)), & x \in \Omega, \rho \in(0,1), t>0 .
\end{array}
$$

Then, we have

$$
\begin{array}{r}
\tau(t) z_{1}^{\prime}(x, \rho, t)+\left(1-\rho \tau^{\prime}(t)\right) \frac{\partial}{\partial \rho} z_{1}(x, \rho, t)=0, \\
\operatorname{in} \Omega \times(0,1) \times(0,+\infty) .
\end{array}
$$

Similarly, we have

$$
\begin{gathered}
\tau(t) z_{2}^{\prime}(x, \rho, t)+\left(1-\rho \tau^{\prime}(t)\right) \frac{\partial}{\partial \rho} z_{2}(x, \rho, t)=0, \\
\operatorname{in} \Omega \times(0,1) \times(0,+\infty) .
\end{gathered}
$$

Therefore, problem (3) is equivalent to 


$$
\begin{cases}\left|u_{t}\right|^{l} u_{t t}+\alpha v-M\left(\|\nabla u\|^{2}\right) \Delta u-\Delta u_{t t}+\int_{0}^{t} h_{1}(t-s) \Delta u(s) \mathrm{d} s-\mu_{1} \Delta z_{1}(x, 1, t)=0, & \text { in } \Omega \times] 0,+\infty[, \\ \left|v_{t}\right|^{l} u_{t t}+\alpha u-M\left(\|\nabla v\|^{2}\right) \Delta v-\Delta v_{t t}+\int_{0}^{t} h_{2}(t-s) \Delta v(s) \mathrm{d} s-\mu_{2} \Delta z_{2}(x, 1, t)=0, & \text { in } \Omega \times] 0,+\infty[, \\ \tau(t) z_{1}^{\prime}(x, \rho, t)+\left(1-\rho \tau^{\prime}(t)\right) \frac{\partial}{\partial \rho} z_{1}(x, \rho, t)=0, & \text { in } \Omega \times(0,1) \times(0,+\infty), \\ \tau(t) z_{2}^{\prime}(x, \rho, t)+\left(1-\rho \tau^{\prime}(t)\right) \frac{\partial}{\partial \rho} z_{2}(x, \rho, t)=0, & \text { in } \Omega \times(0,1) \times(0,+\infty), \\ u(x, t)=v(x, t)=0, & \text { on }[\partial \Omega \times[0, \infty, \\ \left(z_{1}(x, 0, t), z_{2}(x, 0, t)\right)=\left(u_{t}(x, t), v_{t}(x, t)\right), & \text { on } \Omega \times] 0, \infty[, \\ (u(x, 0), v(x, 0))=\left(u_{0}(x), v_{0}(x)\right),\left(u_{t}(x, 0),\left(v_{t}(x, 0)\right)=\left(u_{1}(x), v_{1}(x)\right),\right. & \text { in } \Omega, \\ \left(z_{1}(x, \rho, 0), z_{2}(x, \rho, 0)\right)=\left(f_{0}(x,-\rho \tau(0)), g_{0}(x,-\rho \tau(0))\right), & \text { in } \Omega \times] 0,1[.\end{cases}
$$

To state and prove our result, we need some assumptions.

Assumption 1. Assume that $0<l \leq \gamma$ satisfies

$$
\begin{cases}\gamma \leq \frac{2}{n-2}, & \text { if } n>2, \\ \gamma<\infty, & \text { if } n \leq 2 .\end{cases}
$$

Assumption 2. For the relaxation functions, $h_{i}: \mathrm{IR}_{+} \longrightarrow \mathrm{IR}_{+}$are bounded $C^{1}$ functions such that

$$
a-\int_{0}^{\infty} h_{i}(s) \mathrm{d} s \geq k>0,
$$

and suppose that there exist positive constants $\zeta_{i}$ satisfying

$$
h_{i}^{\prime}(t) \leq-\zeta_{i} h_{i}(t)
$$

for $i=1,2$.

Assumption 3. $\tau$ is a function in $W^{2, \infty}([0, T]), T>0$, such that

$$
\begin{aligned}
E(t)= & \frac{1}{l+2}\left(\left\|u_{t}\right\|_{l+2}^{l+2}+\left\|v_{t}\right\|_{l+2}^{l+2}\right)+2 \alpha \int_{\Omega} u(x, t) v(x, t) \mathrm{d} x \\
& +\frac{b}{2(\gamma+1)}\left(\|\nabla u\|^{2(\gamma+1)}+\|\nabla v\|^{2(\gamma+1)}\right) \\
& +\frac{1}{2}\left(a-\int_{0}^{t} h_{1}(s) \mathrm{d} s\right)\|\nabla u\|^{2}+\frac{1}{2}\left(a-\int_{0}^{t} h_{2}(s) \mathrm{d} s\right) \\
& \cdot\|\nabla v\|^{2}+\frac{1}{2}\left(\left\|\nabla u_{t}\right\|^{2}+\left\|\nabla v_{t}\right\|^{2}\right) \\
& +\frac{1}{2}\left(h_{1} o \nabla u\right)(t)+\frac{1}{2}\left(h_{2} o \nabla v\right)(t) \\
& +\xi \tau(t) \int_{0}^{1}\left(\left\|\nabla z_{1}(x, \rho, t)\right\|^{2}+\left\|\nabla z_{2}(x, \rho, t)\right\|^{2}\right) \mathrm{d} \rho,
\end{aligned}
$$

where $\xi$ is a positive constant such that

$$
\frac{\max \left\{\mu_{1}, \mu_{2}\right\}}{2(1-d)}<\xi
$$

$$
\left(h_{i} o w\right)(t)=\int_{0}^{t} h_{i}(t-s)\|w(., t)-w(., s)\|^{2} \mathrm{~d} s, \quad \text { for } i=1,2 .
$$

$$
\begin{aligned}
0<\tau_{0} \leq \tau^{\prime}(t) & \leq \tau_{1}, \quad \forall t>0, \\
\tau^{\prime}(t) & \leq d<1, \quad \forall t>0 .
\end{aligned}
$$

We define the energy associated to the solution of system (7) by
Theorem 1 (global existence). Let $\left(u_{0}, v_{0}\right) \in\left(H^{2}\right.$ $\left.(\Omega) \cap H_{0}^{1}(\Omega)\right)^{2}$, s, and $\left(f_{0}, g_{0}\right) \in\left(H_{0}^{1}\left(\Omega, H^{1}(0,1)\right)\right)^{2}$ satisfy the compatibility condition

$$
\left(f_{0}(., 0), g_{0}(., 0)\right)=\left(u_{1}, v_{1}\right) .
$$


Assume that Assumptions 1-3 hold. Then, problem (3) admits a weak solution such that $u, v \in L^{\infty}$ $\left(\left(0, \infty ; H^{2}(\Omega) \cap H_{0}^{1}(\Omega)\right), \quad u_{t}, v_{t} \in L^{\infty}\left(0, \infty ; H_{0}^{1}(\Omega)\right)\right.$, and $u_{t t}, v_{t t} \in L^{2}\left(0, \infty, H_{0}^{1}(\Omega)\right)$.

Theorem 2 (decay rates of energy). Assume that Assumptions 1-3 hold. Then, for every $t_{0}>0$ there exist positive constants $K$ and $c^{\prime}$ such that the energy defined by (12) possesses the following decay:

$$
E(t) \leq K e^{-c^{\prime} t}, \quad \forall t \geq t_{0} .
$$

\section{Preliminaries}

Lemma 1 (Sobolev-Poincareinequality). Let $q$ be a number with

$$
2 \leq q<+\infty(n=1,2) \text { or } 2 \leq q \leq \frac{2 n}{(n-2)(n \geq 3)} .
$$

Then, there exists a constant $C_{s}=C_{s}(\Omega, q)$ such that

$$
\|u\|_{q} \leq C_{s}\|\nabla u\|_{2} \text { for } u \in H_{0}^{1}(\Omega) \text {. }
$$

The following lemma states an important property of the convolution operator.

Lemma 2 (see [9]). For $h, \varphi \in C^{1}([0,+\infty[, \mathrm{IR})$, we have

$$
\begin{aligned}
\int_{\Omega} h * \varphi \varphi_{t} \mathrm{~d} x= & -\frac{1}{2} h(t)\|\varphi(t)\|^{2}+\frac{1}{2}\left(h^{\prime} \circ \varphi\right)(t) \\
& -\frac{1}{2} \frac{d}{d t}\left[(h o \varphi)(t)-\left(\int_{0}^{t} h(s) \mathrm{d} s\right)\|\varphi\|^{2}\right] .
\end{aligned}
$$

Lemma 3. Let $\left(u, v, z_{1}, z_{2}\right)$ be a solution of problem (7). Then, the energy functional defined by (12) satisfies

$$
\begin{aligned}
E(t) \leq & -\beta\left(\left\|\nabla z_{1}(x, 1, t)\right\|^{2}+\left\|\nabla z_{2}(x, 1, t)\right\|^{2}\right) \\
& +\lambda\left(\left\|\nabla u_{t}(x, t)\right\|^{2}+\left\|\nabla v_{t}(x, t)\right\|^{2}\right) \\
& +\frac{1}{2}\left[\left(h_{1}^{\prime} o \nabla u\right)(t)+\left(h_{2}^{\prime} o \nabla v\right)(t)\right],
\end{aligned}
$$

where $\lambda=\xi+(\mu / 2), \beta=\xi(1-d)-\mu / 2$, and $\mu=\max \left\{\mu_{1}, \mu_{2}\right\}$ are positive.

Proof. Multiplying the first equation in (7) by $u_{t}$, integrating over $\Omega$, and using integration by parts, we get

$$
\begin{aligned}
& \frac{\mathrm{d}}{\mathrm{d} t}\left[\frac{1}{l+2}\left\|u_{t}\right\|_{l+2}^{l+2}+\frac{b}{2(\gamma+1)}\|\nabla u\|^{2(\gamma+1)}+\frac{1}{2} a\|\nabla u\|^{2}+\frac{1}{2}\left\|\nabla u_{t}\right\|^{2}\right] \\
& \quad-\int_{\Omega} \int_{0}^{t} h_{1}(t-s) \nabla u(s) \nabla u_{t}(t) \mathrm{d} s \mathrm{~d} x \\
& \quad+\alpha \int_{\Omega} u_{t}(x, t) v(x, t) \mathrm{d} x+\mu_{1} \int_{\Omega} \nabla u_{t}(x, t) \nabla z_{1}(x, 1, t) \mathrm{d} x=0 .
\end{aligned}
$$

Consequently, by applying Lemma 2, equation (21) becomes

$$
\begin{aligned}
& \frac{\mathrm{d}}{\mathrm{d} t}\left[\frac{1}{l+2}\left\|u_{t}\right\|_{l+2}^{l+2}+\frac{b}{2(\gamma+1)}\|\nabla u\|^{2(\gamma+1)}+\frac{1}{2}\left(a-\int_{0}^{t} h_{1}(s) \mathrm{d} s\right)\right. \\
& \left.\quad \cdot\|\nabla u\|^{2}+\frac{1}{2}\left\|\nabla u_{t}\right\|^{2}+\frac{1}{2}\left(h_{1} o \nabla u\right)(t)\right] \\
& \quad+\frac{1}{2} h_{1}(t)\|\nabla u(t)\|^{2}-\frac{1}{2}\left(h_{1}^{\prime} o \nabla u\right)(t)+\alpha \int_{\Omega} u_{t}(x, t) v(x, t) \mathrm{d} x \\
& \left.\quad+\mu_{1} \int_{\Omega} \nabla u_{t}(x, t) \nabla z_{1}(x, 1, t)\right) \mathrm{d} x=0 .
\end{aligned}
$$

Similarly by multiplying the second equation in (7) by $v_{t}$, integrating over $\Omega$, and using integration by parts, we get

$$
\begin{aligned}
& \frac{\mathrm{d}}{\mathrm{d} t}\left[\frac{1}{l+2}\left\|v_{t}\right\|_{l+2}^{l+2}+\frac{b}{2(\gamma+1)}\|\nabla v\|^{2(\gamma+1)}+\frac{1}{2}\left(a-\int_{0}^{t} h_{2}(s) \mathrm{d} s\right)\right. \\
& \left.\cdot\|\nabla v\|^{2}+\frac{1}{2}\left\|\nabla v_{t}\right\|^{2}+\frac{1}{2}\left(h_{2} o \nabla v\right)(t)\right] \\
& \quad+\frac{1}{2} h_{2}(t)\|\nabla v(t)\|^{2}-\frac{1}{2}\left(h_{2}^{\prime} o \nabla v\right)(t)+\alpha \int_{\Omega} u(x, t) v_{t}(x, t) \mathrm{d} x \\
& \quad+\mu_{2} \int_{\Omega} \nabla v_{t}(x, t) \nabla z_{2}(x, 1, t) \mathrm{d} x=0 .
\end{aligned}
$$

Multiply the third equation in (7) by $\xi \Delta z_{1}$ and integrate the result over $\Omega \times(0,1)$ to obtain

$$
\begin{aligned}
& \xi \tau(t) \int_{\Omega} \int_{0}^{1} z_{1}^{\prime}(x, \rho, t) \Delta z_{1}(x, \rho, t) \Omega \times(0,1) \mathrm{d} \rho \mathrm{d} x \\
& \quad=-\xi \int_{\Omega} \int_{0}^{1}\left(1-\rho \tau^{\prime}(t)\right) \frac{\partial}{\partial \rho} z_{1}(x, \rho, t) \Delta z_{1}(x, \rho, t) \mathrm{d} \rho \mathrm{d} x
\end{aligned}
$$

Consequently, 


$$
\begin{aligned}
& \frac{\mathrm{d}}{\mathrm{d} t}\left(\xi \tau(t) \int_{\Omega} \int_{0}^{1}\left|\nabla z_{1}(x, \rho, t)\right|^{2} \mathrm{~d} \rho \mathrm{d} x\right) \\
& =\xi \tau^{\prime}(t) \int_{\Omega} \int_{0}^{1}\left|\nabla z_{1}(x, \rho, t)\right|^{2} \mathrm{~d} \rho \mathrm{d} x \\
& \quad-\xi \int_{\Omega} \int_{0}^{1}\left(1-\rho \tau^{\prime}(t)\right) \frac{\partial}{\partial \rho}\left|\nabla z_{1}(x, \rho, t)\right|^{2} \mathrm{~d} \rho \mathrm{d} x \\
& =-\xi \int_{\Omega} \int_{0}^{1} \frac{\partial}{\partial \rho}\left((1-\rho \tau(t))\left|\nabla z_{1}(x, \rho, t)\right|^{2}\right) \mathrm{d} \rho \mathrm{d} x \\
& =-\xi\left(1-\tau^{\prime}(t)\right) \int_{\Omega}\left|\nabla z_{1}(x, 1, t)\right|^{2} \mathrm{~d} x+\xi \int_{\Omega}\left|\nabla u_{t}(x, t)\right|^{2} \mathrm{~d} x .
\end{aligned}
$$

Similarly, we get

$$
\begin{aligned}
& \frac{\mathrm{d}}{\mathrm{d} t}\left(\xi \tau(t) \int_{\Omega} \int_{0}^{1}\left|\nabla z_{2}(x, \rho, t)\right|^{2} \mathrm{~d} \rho \mathrm{d} x\right) \\
& \quad=-\xi\left(1-\tau^{\prime}(t)\right) \int_{\Omega}\left|\nabla z_{2}(x, 1, t)\right|^{2} \mathrm{~d} x+\xi \int_{\Omega}\left|\nabla v_{t}(x, t)\right|^{2} \mathrm{~d} x .
\end{aligned}
$$

Combining (22)-(26), we obtain

$$
\begin{aligned}
E^{\prime}(t)= & -\xi\left(1-\tau^{\prime}(t)\right) \int_{\Omega}\left(\left|\nabla z_{1}(x, 1, t)\right|^{2}+\left|\nabla z_{2}(x, 1, t)\right|^{2}\right) \mathrm{d} x \\
& +\xi \int_{\Omega}\left(\left|\nabla u_{t}(x, t)\right|^{2}+\left|\nabla v_{t}(x, t)\right|^{2}\right) \mathrm{d} x \\
& -\frac{1}{2}\left[h_{1}(t)\|\nabla u(t)\|^{2}+h_{2}(t)\|\nabla v(t)\|^{2}\right] \\
& +\frac{1}{2}\left[\left(h_{1}^{\prime} o \nabla u\right)(t)+\left(h_{2}^{\prime} o \nabla v\right)(t)\right] \\
& -\mu_{1} \int_{\Omega} \nabla u_{t}(x, t) \nabla z_{1}(x, 1, t) \mathrm{d} x \\
& -\mu_{2} \int_{\Omega} \nabla v_{t}(x, t) \nabla z_{2}(x, 1, t) \mathrm{d} x .
\end{aligned}
$$

From Assumption 3, we get

$$
\begin{aligned}
E^{\prime}(t) \leq & -\left(\xi(1-d)-\frac{\mu_{1}}{2}\right) \int_{\Omega}\left|\nabla z_{1}(x, 1, t)\right|^{2} d x \\
& -\left(\xi(1-d)-\frac{\mu_{2}}{2}\right) \int_{\Omega}\left|\nabla z_{2}(x, 1, t)\right|^{2} d x \\
& -\frac{1}{2} h_{1}(t)\left\|\nabla u_{t}(t)\right\|^{2}+\left(\xi+\frac{\mu_{1}}{2}\right)\left\|\nabla u_{t}(t)\right\|^{2} \\
& -\frac{1}{2} h_{2}(t)\|\nabla v(t)\|^{2}+\left(\xi+\frac{\mu_{2}}{2}\right)\left\|\nabla v_{t}(t)\right\|^{2} \\
& +\frac{1}{2}\left[\left(h_{1}^{\prime} o \nabla u\right)(t)+\left(h_{2}^{\prime} o \nabla v\right)(t)\right] .
\end{aligned}
$$

Using (13), this completes the proof.

\section{Global Existence (Proof of Theorem 1)}

Throughout this section we assume $u_{0}, v_{0} \in H^{2}(\Omega) \cap$ $H_{0}^{1}(\Omega), u_{1}, v_{1} \in H_{0}^{1}(\Omega)$, and $f_{0}, g_{0} \in H_{0}^{1}\left(\Omega, H^{1}(0,1)\right)$. We will use the Faedo-Galerkin method to prove the existence of global solutions. Let $T>0$ be fixed and let $w^{k}, k \in I N$ be a basis of $H^{2}(\Omega) \cap H_{0}^{1}(\Omega)$ and $V_{k}$ be the space generated by $w^{k}$. Now, we define, for $1 \leq j \leq k$, the sequence $\phi^{j}(x, \rho)$ as follows:

$$
\phi^{j}(x, 0)=w^{j} .
$$

Then, we may extend $\phi^{j}(x, 0)$ by $\phi^{j}(x, \rho)$ over $L^{2}(\Omega \times$ $(0,1))$ such that $\left(\phi^{j}\right)_{j}$ forms a basis of $L^{2}\left(\Omega, H^{1}(0,1)\right)$ and denote $Z_{k}$ the space generated by $\left\{\phi^{k}\right\}$. We construct approximate solutions $\left(u^{k}, v^{k}, z_{1}^{k}, z_{2}^{k}\right), k=1,2,3, \ldots$, in the form

$$
\begin{aligned}
u^{k}(t) & =\sum_{j=1}^{k} a^{j k}(t) w^{j}, \\
v^{k}(t) & =\sum_{j=1}^{k} b^{j k}(t) w^{j}, \\
z_{1}^{k}(t) & =\sum_{j=1}^{k} c^{j k}(t) \phi^{j}, \\
z_{2}^{k}(t) & =\sum_{j=1}^{k} d^{j k}(t) \phi^{j},
\end{aligned}
$$

where $a^{j k}, b^{j k}, c^{j k}$, and $d^{j k}(j=1,2, \ldots, k)$ are determined by the following ordinary differential equations:

$$
\left\{\begin{array}{l}
\left(\left.\left|u_{t}^{k}\right|\right|^{l} u_{t t}^{k}, w^{j}\right)+\alpha\left(v^{k}, w^{j}\right)+M\left(\left\|\nabla u^{k}(t)\right\|^{2}\right)\left(\nabla u^{k}, \nabla w^{j}\right) \\
\quad+\left(\nabla u_{t t}^{k}, \nabla w^{j}\right)-\int_{0}^{t} h_{1}(t-s)\left(\nabla u^{k}(s), \nabla w^{j}\right) d s \\
\quad+\mu_{1}\left(\nabla z_{1}^{k}(., 1), \nabla w^{j}\right)=0,1 \leq j \leq k \\
\left(\left|v_{t}^{k}\right|^{l} v_{t t}^{k}, w^{j}\right)+\alpha\left(u^{k}, w^{j}\right)+M\left(\left\|\nabla v^{k}(t)\right\|^{2}\right)\left(\nabla v^{k}, \nabla w^{j}\right) \\
\quad+\left(\nabla v_{t t}^{k}, \nabla w^{j}\right)-\int_{0}^{t} h_{2}(t-s)\left(\nabla v^{k}(s), \nabla w^{j}\right) d s \\
\quad+\mu_{2}\left(\nabla z_{2}^{k}(., 1), \nabla w^{j}\right)=0,1 \leq j \leq k \\
z_{1}^{k}(x, 0, t)=u_{t}^{k}(x, t), z_{2}^{k}(x, 0, t)=v_{t}^{k}(x, t),
\end{array}\right.
$$

$$
u^{k}(0)=u_{0}^{k}=\sum_{j=1}^{k}\left(u_{0}, w^{j}\right) w^{j} \longrightarrow u_{0}, \text { in } H^{2}(\Omega) \cap H_{0}^{1}(\Omega) \text { as } k
$$




$$
\begin{aligned}
& v^{k}(0)=v_{0}^{k}=\sum_{j=1}^{k}\left(v_{0}, w^{j}\right) w^{j} \longrightarrow v_{0}, \\
& \text { in } H^{2}(\Omega) \cap H_{0}^{1}(\Omega) \text { as } k \longrightarrow+\infty \text {, } \\
& u_{t}^{k}(0)=u_{1}^{k}=\sum_{j=1}^{k}\left(u_{1}, w^{j}\right) w^{j} \longrightarrow u_{1} \\
& \text { in } H_{0}^{1}(\Omega) \text { as } k \longrightarrow+\infty \text {, } \\
& v_{t}^{k}(0)=v_{1}^{k}=\sum_{j=1}^{k}\left(v_{1}, w^{j}\right) w^{j} \longrightarrow v_{1}, \\
& \text { in } H_{0}^{1}(\Omega) \text { as } k \longrightarrow+\infty \text {, } \\
& \begin{cases}\left(\tau(t) \frac{\partial}{\partial t} z_{1}^{k}+\left(1-\rho \tau^{\prime}(t)\right) \frac{\partial}{\partial \rho} z_{1}^{k}, \phi^{j}\right)=0, & 1 \leq j \leq k, \\
\left(\tau(t) \frac{\partial}{\partial t} z_{2}^{k}+\left(1-\rho \tau^{\prime}(t)\right) \frac{\partial}{\partial \rho} t z_{2}^{k} n, q \phi^{j}\right)=0, & 1 \leq j \leq k,\end{cases} \\
& z_{1}^{k}(\rho, 0)=\sum_{j=1}^{k}\left(f_{0}, \phi^{j}\right) \phi^{j} \longrightarrow f_{0}, \\
& \text { in } H_{0}^{1}\left(\Omega, H^{1}(0,1)\right) \text { as } k \longrightarrow+\infty \text {, } \\
& z_{2}^{k}(\rho, 0)=\sum_{j=1}^{k}\left(g_{0}, \phi^{j}\right) \phi^{j} \longrightarrow g_{0}, \\
& \text { in } H_{0}^{1}\left(\Omega, H^{1}(0,1)\right) \text { as } k \longrightarrow+\infty \text {. }
\end{aligned}
$$

Noting that $l / 2(l+1)+1 / 2(l+1)+1 / 2=1$, from the generalized Hölder inequality, we obtain

$$
\begin{aligned}
\left(\left|u_{t}^{k}\right|^{l} u_{t t}^{k}, w_{j}\right)= & \int_{\Omega}\left|u_{t}^{k}\right|^{l} u_{t t}^{k} w_{j} \mathrm{~d} x \leq\left(\int_{\Omega}\left|u_{t}^{k}\right|^{2(l+1)} \mathrm{d} x\right)^{l /(2(l+1))} \\
& \cdot\left\|u_{t t}^{k}\right\|_{2(l+1)}\left\|w_{j}\right\|_{2} .
\end{aligned}
$$

Since Assumption 2 holds, according to Sobolev embedding, the nonlinear terms $\left(\left|u_{t}^{k}\right|^{l} u_{t t}^{k}, w_{j}\right)$ and $\left(\left|v_{t}^{k}\right|^{l} v_{t t}^{k}, w_{j}\right)$ in (31) make sense.

The standard theory of ODE guarantees that systems (31)-(38) have a unique solution in $\left[0, t_{k}\right)$, with $0<t_{k}<T$. In the next step, we obtain a priori estimates for the solution of systems (31)-(38), so that it can be extended outside $\left[0, t_{k}\right)$ to obtain one solution defined for all $t>0$, using a standard compactness argument for the limiting procedure.

3.1. First Estimate. Since the sequences $u_{0}^{k}, v_{0}^{k}, u_{1}^{k}, v_{1}^{k}, z_{1}^{k}(\rho, 0)$, and $z_{2}^{k}(\rho, 0)$ converge and from Lemma 3 with employing Gronwall's lemma, we can find a positive constant $C_{1}$ independent of $k$ such that

$$
E^{k}(t)+\beta \int_{0}^{t}\left(\left\|\nabla z_{1}^{k}(x, 1, s)\right\|^{2}+\left\|\nabla z_{2}^{k}(x, 1, s)\right\|^{2}\right) \mathrm{d} s \leq C_{1},
$$

where

$$
\begin{aligned}
E^{k}(t)= & \frac{1}{l+2}\left(\left\|u_{t}^{k}\right\|_{l+2}^{l+2}+\left\|v_{t}^{k}\right\|_{l+2}^{l+2}\right)+2 \alpha \int_{\Omega} u^{k} v^{k} \mathrm{~d} x \\
& +\frac{b}{2(\gamma+1)}\left(\left\|\nabla u^{k}\right\|^{2(\gamma+1)}+\left\|\nabla v^{k}\right\|^{2(\gamma+1)}\right) \\
& +\frac{1}{2}\left(a-\int_{0}^{t} h_{1}(s) \mathrm{d} s\right)\left\|\nabla u^{k}\right\|^{2} \\
& +\frac{1}{2}\left(a-\int_{0}^{t} h_{2}(s) \mathrm{d} s\right)\left\|\nabla v^{k}\right\|^{2}+\frac{1}{2}\left(\left\|\nabla u_{t}^{k}\right\|^{2}+\left\|\nabla v_{t}^{k}\right\|^{2}\right) \\
& +\frac{1}{2}\left[\left(h_{1} o \nabla u^{k}\right)(t)+\left(h v_{2} o \nabla v^{k}\right)(t)\right] \\
& +\xi \tau(t) \int_{0}^{1}\left(\left\|\nabla z_{1}^{k}(x, \rho, t)\right\|\right)^{2}+\left(\left\|\nabla z_{2}^{k}(x, \rho, t)\right\|\right)^{2} \mathrm{~d} \rho .
\end{aligned}
$$

Noting Assumption 1 and estimate (40) yields that

$u^{k}, v^{k}$ are bounded in $L_{\mathrm{loc}}^{\infty}\left(0, \infty, H_{0}^{1}(\Omega)\right)$,

$u_{t}^{k}, v_{t}^{k}$ are bounded in $L_{\mathrm{loc}}^{\infty}\left(0, \infty, H_{0}^{1}(\Omega)\right)$,

$z_{1}^{k}(x, \rho, t), z_{2}^{k}(x, \rho, t)$ are bounded in $L_{\mathrm{loc}}^{\infty}\left(0, \infty, L^{1}\left(0,1, H_{0}^{1}(\Omega)\right)\right)$.

3.2. Second Estimate. Multiplying the first equation (respectively, the second equation) in (31) by $a_{t t}^{j k}$ (respectively, by $b_{t t}^{j k}$ ) and summing over $j$ from 1 to $k$, it follows that

$$
\left\{\begin{array}{l}
\int_{\Omega}\left|u_{t}^{k}\right|^{l}\left|u_{t t}^{k}\right|^{2} \mathrm{~d} x-\alpha \int_{\Omega} v_{t}^{k} u_{t}^{k} \mathrm{~d} x+\int_{\Omega} M\left(\left\|\nabla u^{k}\right\|^{2}\right) \nabla u^{k} \nabla u_{t t}^{k} \mathrm{~d} x \\
+\left\|\nabla u_{t t}^{k}\right\|^{2}=\int_{0}^{t} h_{1}(t-s) \int_{\Omega} \nabla u^{k}(s) \nabla u_{t t}^{k}(t) \mathrm{d} x \mathrm{~d} s \\
\quad-\mu_{1} \int_{\Omega} \nabla u_{t t}^{k} \nabla\left(z_{1}^{k}(x, 1, t)\right) \mathrm{d} x \\
\int_{\Omega}\left|v_{t}^{k}\right|^{l}\left|v_{t t}^{k}\right|^{2} \mathrm{~d} x-\alpha \int_{\Omega} v_{t}^{k} u_{t}^{k} \mathrm{~d} x+\int_{\Omega} M\left(\left\|\nabla v^{k}\right\|^{2}\right) \nabla v^{k} \nabla v_{t t}^{k} \mathrm{~d} x \\
+\left\|\nabla v_{t t}^{k}\right\|^{2}=\int_{0}^{t} h_{2}(t-s) \int_{\Omega} \nabla v^{k}(s) \nabla v_{t t}^{k}(t) \mathrm{d} x \mathrm{~d} s \\
\quad-\mu_{2} \int_{\Omega} \nabla v_{t t}^{k} \nabla\left(z_{2}^{k}(x, 1, t)\right) \mathrm{d} x .
\end{array}\right.
$$

Differentiating (36) with respect to $t$, we get 


$$
\left\{\begin{array}{l}
\left(\left(\frac{\tau(t)}{\left(1-\rho \tau^{\prime}(t)\right)}\right)^{\prime} \frac{\partial}{\partial t} z_{1}^{k}+\frac{\tau(t)}{\left(1-\rho \tau^{\prime}(t)\right)} \frac{\partial^{2}}{\partial t^{2}} z_{1}^{k}+\frac{\partial^{2}}{\partial t \partial \rho} z_{1}^{k}, \phi^{j}\right)=0 \\
\left(\left(\frac{\tau(t)}{\left(1-\rho \tau^{\prime}(t)\right)}\right)^{\prime} \frac{\partial}{\partial t} z_{2}^{k}+\frac{\tau(t)}{\left(1-\rho \tau^{\prime}(t)\right)} \frac{\partial^{2}}{\partial t^{2}} z_{2}^{k}+\frac{\partial^{2}}{\partial t \partial \rho} z_{2}^{k}, \phi^{j}\right)=0
\end{array}\right.
$$

Multiplying the first equation by $c_{t}^{j k}$ (respectively, the second equation by $d_{t}^{j k}$ ) and summing over $j$ from 1 to $k$, it follows that

$$
\left\{\begin{array}{l}
\left(\frac{\tau(t)}{\left(1-\rho \tau^{\prime}(t)\right)}\right)^{\prime}\left\|\frac{\partial}{\partial t} z_{1}^{k}\right\|^{2}+\frac{1}{2} \frac{\tau(t)}{\left(1-\rho \tau^{\prime}(t)\right)} \frac{\mathrm{d}}{\mathrm{d} t}\left\|\frac{\partial}{\partial t} z_{1}^{k}\right\|^{2}+\frac{1}{2} \frac{\mathrm{d}}{\mathrm{d} \rho}\left\|\frac{\partial}{\partial t} z_{1}^{k}\right\|^{2}=0, \\
\left(\frac{\tau(t)}{\left(1-\rho \tau^{\prime}(t)\right)}\right)^{\prime}\left\|\frac{\partial}{\partial t} z_{2}^{k}\right\|^{2}+\frac{1}{2} \frac{\tau(t)}{\left(1-\rho \tau^{\prime}(t)\right)} \frac{\mathrm{d}}{\mathrm{d} t}\left\|\frac{\partial}{\partial t} z_{2}^{k}\right\|^{2}+\frac{1}{2} \frac{\mathrm{d}}{\mathrm{d} \rho}\left\|\frac{\partial}{\partial t} z_{2}^{k}\right\|^{2}=0,
\end{array}\right.
$$

and then we have

$$
\left\{\begin{array}{l}
\frac{1}{2}\left(\frac{\tau(t)}{\left(1-\rho \tau^{\prime}(t)\right)}\right)^{\prime}\left\|\frac{\partial}{\partial t} z_{1}^{k}\right\|^{2}+\frac{1}{2} \frac{\mathrm{d}}{\mathrm{d} t}\left(\frac{\tau(t)}{\left(1-\rho \tau^{\prime}(t)\right)}\left\|\frac{\partial}{\partial t} z_{1}^{k}\right\|^{2}\right) \\
+\frac{1}{2} \frac{\mathrm{d}}{\mathrm{d} \rho}\left\|\frac{\partial}{\partial t} z_{1}^{k}\right\|^{2}=0, \\
\frac{1}{2}\left(\frac{\tau(t)}{\left(1-\rho \tau^{\prime}(t)\right)}\right)^{\prime}\left\|\frac{\partial}{\partial t} z_{2}^{k}\right\|^{2}+\frac{1}{2} \frac{\mathrm{d}}{\mathrm{d} t}\left(\frac{\tau(t)}{\left(1-\rho \tau^{\prime}(t)\right)}\left\|\frac{\partial}{\partial t} z_{2}^{k}\right\|^{2}\right) \\
+\frac{1}{2} \frac{\mathrm{d}}{\mathrm{d} \rho}\left\|\frac{\partial}{\partial t} z_{2}^{k}\right\|^{2}=0 .
\end{array}\right.
$$

Integrating over $(0,1)$ with respect to $\rho$, we obtain

$$
\left\{\begin{array}{l}
\frac{1}{2} \int_{0}^{1}\left(\frac{\tau(t)}{\left(1-\rho \tau^{\prime}(t)\right)}\right)^{\prime}\left\|\frac{\partial}{\partial t} z_{1}^{k}\right\|^{2} \mathrm{~d} \rho+\frac{1}{2} \frac{\mathrm{d}}{\mathrm{d} t}\left(\int_{0}^{1} \frac{\tau(t)}{\left(1-\rho \tau^{\prime}(t)\right)}\left\|\frac{\partial}{\partial t} z_{1}^{k}\right\|^{2} \mathrm{~d} \rho\right)+\frac{1}{2}\left\|\frac{\partial}{\partial t} z_{1}^{k}(x, 1, t)\right\|^{2}-\frac{1}{2}\left\|u_{t t}^{k}(x, t)\right\|^{2}=0, \\
\frac{1}{2} \int_{0}^{1}\left(\frac{\tau(t)}{\left(1-\rho \tau^{\prime}(t)\right)}\right)^{\prime}\left\|\frac{\partial}{\partial t} z_{2}^{k}\right\|^{2} \mathrm{~d} \rho+\frac{1}{2} \frac{\mathrm{d}}{\mathrm{d} t}\left(\int_{0}^{1} \frac{\tau(t)}{\left(1-\rho \tau^{\prime}(t)\right)}\left\|\frac{\partial}{\partial t} z_{2}^{k}\right\|^{2} \mathrm{~d} \rho\right)+\frac{1}{2}\left\|\frac{\partial}{\partial t} z_{2}^{k}(x, 1, t)\right\|^{2}-\frac{1}{2}\left\|v_{t t}^{k}(x, t)\right\|^{2}=0 .
\end{array}\right.
$$

Summing (43) and (47) and as $M(r) \geq a$, we get

$$
\left\{\begin{array}{l}
\int_{\Omega}\left|u_{t}^{k}\right|^{l}\left|u_{t t}^{k}\right|^{2} d x+\left\|\nabla u_{t t}^{k}\right\|^{2}+\frac{1}{2} \frac{\mathrm{d}}{\mathrm{d} t}\left(\int_{0}^{1} \frac{\tau(t)}{\left(1-\rho \tau^{\prime}(t)\right)}\left\|\frac{\partial}{\partial t} z_{1}^{k}\right\|^{2} \mathrm{~d} \rho\right)+\frac{1}{2}\left\|\frac{\partial}{\partial t} z_{1}^{k}(x, 1, t)\right\|^{2} \leq-a \int_{\Omega} \nabla u^{k} \nabla u_{t t}^{k} \mathrm{~d} x+\frac{1}{2}\left\|u_{t t}^{k}(x, t)\right\|^{2} \\
+\alpha \int_{\Omega} v_{t}^{k} u_{t}^{k} \mathrm{~d} x-\frac{1}{2} \int_{0}^{1}\left(\frac{\tau(t)}{\left(1-\rho \tau^{\prime}(t)\right)}\right)^{\prime}\left\|\frac{\partial}{\partial t} z_{1}^{k}\right\|^{2} \mathrm{~d} \rho+\int_{0}^{t} h_{1}(t-s) \int_{\Omega} \nabla u^{k}(s) \nabla u_{t t}^{k}(t) \mathrm{d} x \mathrm{~d} s-\mu_{1} \int_{\Omega} \nabla u_{t t}^{k} \nabla z_{1}^{k}(x, 1, t) \mathrm{d} x, \\
\int_{\Omega}\left|v_{t}^{k}\right|^{l}\left|v_{t t}^{k}\right|^{2} \mathrm{~d} x+\left\|\nabla v_{t t}^{k}\right\|^{2}+\frac{1}{2} \frac{\mathrm{d}}{\mathrm{d} t}\left(\int_{0}^{1} \frac{\tau(t)}{\left(1-\rho \tau^{\prime}(t)\right)}\left\|\frac{\partial}{\partial t} z_{2}^{k}\right\|^{2} \mathrm{~d} \rho\right)+\frac{1}{2}\left\|\frac{\partial}{\partial t} z_{2}^{k}(x, 1, t)\right\|^{2} \leq-a \int_{\Omega} \nabla v^{k} \nabla v_{t t}^{k} \mathrm{~d} x+\frac{1}{2}\left\|v_{t t}^{k}(x, t)\right\|^{2} \\
+\alpha \int_{\Omega} v_{t}^{k} u_{t}^{k} \mathrm{~d} x-\frac{1}{2} \int_{0}^{1}\left(\frac{\tau(t)}{\left(1-\rho \tau^{\prime}(t)\right)}\right)^{\prime}\left\|\frac{\partial}{\partial t} z_{2}^{k}\right\|^{2} \mathrm{~d} \rho+\int_{0}^{t} h_{2}(t-s) \int_{\Omega} \nabla v^{k}(s) \nabla v_{t t}^{k}(t) \mathrm{d} x \mathrm{~d} s-\mu_{2} \int_{\Omega} \nabla v_{t t}^{k} \nabla z_{2}^{k}(x, 1, t) \mathrm{d} x .
\end{array}\right.
$$


By Young's inequality, the right hand side of (48) can be estimated as follows:

$$
\left\{\begin{array}{l}
\left|\int_{\Omega} a \nabla u^{k} \nabla u_{t t}^{k} \mathrm{~d} x\right| \leq \eta\left\|\nabla u_{t t}^{k}\right\|^{2}+\frac{a^{2}}{4 \eta}\left\|\nabla u^{k}\right\|^{2}, \\
\left|\int_{\Omega} a \nabla v^{k} \nabla v_{t t}^{k} \mathrm{~d} x\right| \leq \eta\left\|\nabla v_{t t}^{k}\right\|^{2}+\frac{a^{2}}{4 \eta}\|\| \nabla v^{k} \|^{2} .
\end{array}\right.
$$

Using Cauchy-Schwarz inequality and Sobolev-Poincare inequality, we obtain

$$
\begin{aligned}
& \alpha \int_{\Omega} v_{t}^{k} u_{t}^{k} \mathrm{~d} x \leq \frac{\alpha}{2}\left(\left\|v_{t}^{k}\right\|^{2}+\left\|u_{t}^{k}\right\|^{2}\right) \leq \frac{C_{s} \alpha}{2}\left(\left\|\nabla v_{t}^{k}\right\|^{2}+\left\|\nabla u_{t}^{k}\right\|^{2}\right) \\
& \left|\int_{0}^{t} h_{1}(t-s) \int_{\Omega} \nabla u^{k}(s) \nabla u_{t t}^{k}(t) \mathrm{d} x \mathrm{~d} s\right| \leq \eta\left\|\nabla u_{t t}^{k}\right\|^{2} \\
& \quad+\frac{1}{4 \eta} \int_{\Omega}\left(\int_{0}^{t} h_{1}(t-s) \nabla u^{k}(s) \mathrm{d} s \mathrm{~d} x\right)^{2} \\
& \leq \eta\left\|\nabla u_{t t}^{k}\right\|^{2}+\frac{1}{4 \eta} \int_{0}^{t} h(s) \mathrm{d} s \int_{\Omega} \int_{0}^{t} h_{1}(t-s)\left|\nabla u^{k}(s)\right|^{2} \mathrm{~d} s \mathrm{~d} x \\
& \leq \eta\left\|\nabla u_{t t}^{k}\right\|^{2}+\frac{1}{4 \eta}(a-k) \int_{\Omega} \int_{0}^{t} h_{1}(t-s)\left|\nabla u^{k}(s)\right|^{2} \mathrm{~d} s \mathrm{~d} x \\
& \leq \eta\left\|\nabla u_{t t}^{k}\right\|^{2}+\frac{1}{4 \eta}(a-k) \int_{0}^{t} h_{1}(t-s)\left\|\nabla u^{k}(s)\right\|^{2} \mathrm{~d} s \\
& \leq \eta\left\|\nabla u_{t t}^{k}\right\|^{2}+\frac{1}{4 \eta}(a-k) h_{1}(0) \int_{0}^{t}\left\|\nabla u^{k}(s)\right\|^{2} \mathrm{~d} s .
\end{aligned}
$$

Similarly,

$$
\begin{gathered}
\left|\int_{0}^{t} h_{2}(t-s) \int_{\Omega} \nabla v^{k}(s) \nabla v^{k_{t t}}(t) \mathrm{d} x \mathrm{~d} s\right| \leq \eta\left\|\nabla v^{k_{t t}}\right\|^{2} \\
\quad+\frac{1}{4 \eta}(a-k) h_{2}(0) \int_{0}^{t}\left(\nabla v^{k}(s)\right)^{2} \mathrm{~d} s, \\
\left\{\begin{array}{l}
\left|\mu_{1} \int_{\Omega} \nabla u_{t t}^{k} \nabla z_{1}^{k}(x, 1, t) \mathrm{d} x\right| \leq \eta \mu_{1}^{2}\left\|\nabla u_{t t}^{k}\right\|^{2}+\frac{1}{4 \eta}\left\|\nabla z_{1}^{k}(x, 1, t)\right\|^{2}, \\
\left|\mu_{2} \int_{\Omega} \nabla v_{t t}^{k} \nabla z_{2}^{k}(x, 1, t) \mathrm{d} x\right| \leq \eta \mu_{2}^{2}\left\|\nabla v_{t t}^{k}\right\|^{2}+\frac{1}{4 \eta}\left\|\nabla z_{2}^{k}(x, 1, t)\right\|^{2} .
\end{array}\right.
\end{gathered}
$$

By using (49)-(53) in (48), we deduce

$$
\begin{aligned}
& \int_{\Omega}\left|u_{t}^{k}\right|^{l}\left|u_{t t}^{k}\right|^{2} \mathrm{~d} x+\left\|\nabla u_{t t}^{k}\right\|^{2}+\frac{1}{2} \frac{\mathrm{d}}{\mathrm{d} t}\left(\int_{0}^{1} \frac{\tau(t)}{1-\rho \tau^{\prime}(t)}\left\|\frac{\partial}{\partial t} z_{1}^{k}\right\|^{2} \mathrm{~d} \rho\right) \\
& +\frac{1}{2}\left\|\frac{\partial}{\partial t} z_{1}^{k}(x, 1, t)\right\|^{2} \leq\left(\eta\left(\mu_{1}^{2}+2\right)+\frac{C_{s}^{2}}{2}\right)\left\|\nabla u_{t t}^{k}\right\|^{2} \\
& +\frac{C_{s} \alpha}{2}\left(\left\|\nabla v_{t}^{k}\right\|^{2}+\left\|\nabla u_{t}^{k}\right\|^{2}\right)+\frac{a^{2}}{4 \eta}\left\|\nabla u^{k}\right\|^{2}+\frac{1}{4 \eta}\left\|\nabla z_{1}^{k}(x, 1, t)\right\|^{2} \\
& +\frac{1}{4 \eta}(a-k) h_{1}(0) \int_{0}^{t}\left\|\nabla u^{k}(s)\right\|^{2} \mathrm{~d} s \\
& -\frac{1}{2} \int_{0}^{1}\left(\frac{\tau(t)}{1-\rho \tau^{\prime}(t)}\right)^{\prime}\left\|\frac{\partial}{\partial t} z_{1}^{k}\right\|^{2} \mathrm{~d} \rho \\
& \int_{\Omega}\left|v_{t}^{k}\right|^{l}\left|v_{t t}^{k}\right|^{2} \mathrm{~d} x+\left\|\nabla v_{t t}^{k}\right\|^{2}+\frac{1}{2} \frac{\mathrm{d}}{\mathrm{d} t}\left(\int_{0}^{1} \frac{\tau(t)}{1-\rho \tau^{\prime}(t)}\left\|\frac{\partial}{\partial t} z_{2}^{k}\right\|^{2} \mathrm{~d} \rho\right) \\
& +\frac{1}{2}\left\|\frac{\partial}{\partial t} z_{2}^{k}(x, 1, t)\right\|^{2} \leq\left(\eta\left(\mu_{2}^{2}+2\right)+\frac{C_{s}^{2}}{2}\right)\left\|\nabla v_{t t}^{k}\right\|^{2} \\
& +\frac{C_{s} \alpha}{2}\left(\left\|\nabla v_{t}^{k}\right\|^{2}+\left\|\nabla u_{t}^{k}\right\|^{2}\right)+\frac{a^{2}}{4 \eta}\left\|\nabla v^{k}\right\|^{2}+\frac{1}{4 \eta}\left\|\nabla z_{2}^{k}(x, 1, t)\right\|^{2} \\
& +\frac{1}{4 \eta}(a-k) h_{2}(0) \int_{0}^{t}\left\|\nabla v^{k}(s)\right\|^{2} \mathrm{~d} s \\
& -\frac{1}{2} \int_{0}^{1}\left(\frac{\tau(t)}{1-\rho \tau^{\prime}(t)}\right)^{\prime}\left\|\frac{\partial}{\partial t} z_{2}^{k}\right\|^{2} \mathrm{~d} \rho .
\end{aligned}
$$

By using Assumption 3 and taking the first estimate (40) into account, we infer 


$$
\left\{\begin{array}{l}
\int_{\Omega}\left|u_{t}^{k}\right|^{l}\left|u_{t t}^{k}\right|^{2} \mathrm{~d} x+\left(1-\left(\eta\left(\mu_{1}^{2}+2\right)+\frac{C_{s}^{2}}{2}\right)\right)\left\|\nabla u_{t t}^{k}\right\|^{2} \\
+\frac{1}{2} \frac{\mathrm{d}}{\mathrm{d} t}\left(\int_{0}^{1} \frac{\tau(t)}{1-\rho \tau^{\prime}(t)}\left\|\frac{\partial}{\partial t} z_{1}^{k}\right\|^{2} \mathrm{~d} \rho\right) \\
+\frac{1}{2}\left\|\frac{\partial}{\partial t} z_{1}^{k}(x, 1, t)\right\|^{2} \leq C_{2}+\frac{1}{4 \eta}\left(a-k_{1}\right) h_{1}(0) C_{1} T \\
+a_{1} \int_{0}^{1} \frac{\tau(t)}{1-\rho \tau^{\prime}(t)}\left\|\frac{\partial}{\partial t} z_{1}^{k}\right\|^{2} \mathrm{~d} \rho, \\
\int_{\Omega}\left|v_{t}^{k}\right|^{l}\left|v_{t t}^{k}\right|^{2} \mathrm{~d} x+\left(1-\left(\eta\left(\mu_{2}^{2}+2\right)+\frac{C_{s}^{2}}{2}\right)\right)\left\|\nabla v_{t t}^{k}\right\|^{2} \\
+\frac{1}{2} \frac{\mathrm{d}}{\mathrm{d} t}\left(\int_{0}^{1} \frac{\tau(t)}{1-\rho \tau^{\prime}(t)}\left\|\frac{\partial}{\partial t} z_{2}^{k}\right\|^{2} \mathrm{~d} \rho\right) \\
+\frac{1}{2}\left\|\frac{\partial}{\partial t} z_{2}^{k}(x, 1, t)\right\|^{2} \leq C_{2}+\frac{1}{4 \eta}\left(a-k_{2}\right) h_{2}(0) C_{1} T \\
+a_{2} \int_{0}^{1} \frac{\tau(t)}{1-\rho \tau^{\prime}(t) \|}\left\|\frac{\partial}{\partial t} z_{2}^{k}\right\|^{2} \mathrm{~d} \rho,
\end{array}\right.
$$

where $C_{2}$ is a positive constant which depends on $\eta, a, C_{1}, C_{s}, \alpha$.

Integrating (55) over $(0, t)$, we obtain

$$
\left\{\begin{array}{l}
\int_{0}^{t}\left|u_{t}^{k}\right|^{l}\left|u_{t t}^{k}\right|^{2} \mathrm{~d} x \mathrm{~d} t+\left(1-\left(\eta\left(\mu_{1}^{2}+2\right)+\frac{C_{s}^{2}}{2}\right)\right) \\
\int_{0}^{t}\left\|\nabla u_{t t}^{k}(s)\right\|^{2} d s+\int_{0}^{1} \frac{\tau(t)}{1-\rho \tau^{\prime}(t)}\left\|\frac{\partial}{\partial t} z_{1}^{k}\right\|^{2} \mathrm{~d} \rho \\
+\frac{1}{2} \int_{0}^{1}\left\|\frac{\partial}{\partial t} z_{1}^{k}(x, 1, t)\right\|^{2} \mathrm{~d} t \leq C_{2} T+\frac{1}{4 \eta}(a-k) h_{1}(0) C_{1} T^{2} \\
+a_{1} \int_{0}^{t} \int_{0}^{1} \frac{\tau(t)}{1-\rho \tau^{\prime}(t)}\left\|\frac{\partial}{\partial s} z_{1}^{k}\right\|^{2} \mathrm{~d} \rho \mathrm{d} s, \\
\int_{0}^{t} \int_{\Omega}\left|v_{t}^{k}\right|^{l}\left|v_{t t}^{k}\right|^{2} \mathrm{~d} x \mathrm{~d} t+\left(1-\left(\eta\left(\mu_{2}^{2}+2\right)+\frac{C_{s}^{2}}{2}\right)\right) \\
\int_{0}^{t}\left\|\nabla v_{t t}^{k}(s)\right\|^{2} \mathrm{~d} s+\int_{0}^{1} \frac{\tau(t)}{1-\rho \tau^{\prime}(t)}\left\|\frac{\partial}{\partial t} z_{2}^{k}\right\|^{2} \mathrm{~d} \rho \\
+\frac{1}{2} \int_{0}^{t}\left\|\frac{\partial}{\partial t} z_{2}^{k}(x, 1, t)\right\|^{2} d t \leq C_{2} T+\frac{1}{4 \eta}(a-k) h_{2}(0) C_{1} T^{2} \\
++a_{2} \int_{0}^{t} \int_{0}^{1} \frac{\tau(s)}{1-\rho \tau^{\prime}(s)}\left\|\frac{\partial}{\partial s} z_{2}^{k}\right\| \|^{2} \mathrm{~d} \rho \mathrm{d} s .
\end{array}\right.
$$

For a suitable $\eta>0$ such that $\left.1-\left(\eta\left(\mu_{i}^{2}+2\right) t+n C_{s}^{2} / 2\right)\right)>0$ for $i=1,2$ and using Gronwall's lemma, we obtain the second estimate

$$
\begin{gathered}
\int_{0}^{t}\left(\left\|\nabla u^{k_{t t}}(s)\right\|^{2}+\left\|\nabla v^{k_{t t}}(s)\right\|^{2}\right) \mathrm{d} s+\int_{0}^{1} \frac{\tau(t)}{1-\rho \tau^{\prime}(t)} \\
\cdot\left(\left\|\frac{\partial}{\partial t} z_{1}^{k}\right\|^{2}+\left\|\frac{\partial}{\partial t} z_{2}^{k}\right\|^{2}\right) \mathrm{d} \rho \leq C_{3} .
\end{gathered}
$$

We observe from estimates (40) and (57) that there exist subsequences $\left(u^{m}\right)$ of $\left(u^{k}\right)$ and $\left(v^{m}\right)$ of $\left(v^{k}\right)$ such that

$$
\left(u^{m}, v^{m}\right) \longrightarrow(u, v) \text { weakly star in } L^{\infty}\left(0, T, H_{0}^{1}(\Omega)\right),
$$

$\left.\left(u_{t}^{m}, v_{t}^{m}\right) \longrightarrow\left(u_{t}, v_{t}\right)\right)$ weakly star in $L^{\infty}\left(0, T, H_{0}^{1}(\Omega)\right)$,

$\left(u_{t t}^{m}, v_{t t}^{m}\right) \longrightarrow\left(u_{t t}, v_{t t}\right)$ weakly in $L^{2}\left(0, T, H_{0}^{1}(\Omega)\right)$,

$\left(z_{1}^{m}, z_{1}^{m}\right) \longrightarrow\left(z_{1}, z_{2}\right)$ weakly starin $L^{\infty}\left(0, T, H_{0}^{1}\left(\Omega, L^{2}(0,1)\right)\right)$,

$$
\begin{aligned}
&\left(\frac{\partial}{\partial t} z_{1}^{m}, \frac{\partial}{\partial t} z_{2}^{m}\right) \rightarrow\left(\frac{\partial}{\partial t} z_{1}, \frac{\partial}{\partial t} z_{2}\right) \text { weaklystarin } \\
& \cdot L^{\infty}\left(0, T, L^{2}(\Omega \times(0,1))\right),
\end{aligned}
$$

In the following, we will treat the nonlinear term. From the first estimate (40) and Lemma 1, we deduce

$$
\begin{aligned}
\left\|\left|u_{t}^{k}\right|^{l} u_{t}^{k}\right\|_{L^{2}\left(0, T, L^{2}(\Omega)\right)} & =\int_{0}^{T}\left\|u_{t}^{k}\right\|_{2(l+1)}^{2(l+1)} \mathrm{d} t \\
& \leq C_{s}^{2(l+1)} \int_{0}^{T}\left\|\nabla u_{t}^{k}\right\|_{2}^{2(l+1)} d t \leq C_{s}^{2(l+1)} C_{1}^{(l+1)} T .
\end{aligned}
$$

On the other hand, from Aubin-Lions theorem (see [10]), we deduce that there exists a subsequence of $\left(u^{m}\right)$, still denoted by $\left(u^{m}\right)$ such that

$$
u_{t}^{m} \longrightarrow u_{t} \text { strongly in } L^{2}\left(0, T, L^{2}(\Omega)\right),
$$

which implies

$$
u_{t}^{m} \longrightarrow u_{t} \text { almost everywhere in } \mathscr{A} \text {. }
$$

Hence,

$$
\left|u_{t}^{m}\right|^{l} u_{t}^{m} \longrightarrow\left|u_{t}\right|^{l} u_{t} \text { almost everywhere in } \mathscr{A},
$$

where $\mathscr{A}=\Omega \times(0, T)$. Thus, using (64) and (66) and Lions lemma, we derive

$$
\left|u_{t}^{m}\right|^{l} u_{t}^{m} \longrightarrow\left|u_{t}\right|^{l} u_{t} \text { weakly in } L^{2}\left(0, T, L^{2}(\Omega)\right) .
$$

Similarly,

$$
\begin{gathered}
\left|v_{t}^{m}\right|^{l} v_{t}^{m} \longrightarrow\left|v_{t}\right|^{l} v_{t} \text { weakly in } L^{2}\left(0, T, L^{2}(\Omega)\right), \\
\left(z_{1}^{m}, z_{2}^{m}\right) \longrightarrow\left(z_{1}, z_{2}\right) \text { strongly in } L^{2}\left(0, T, L^{2}(\Omega)\right),
\end{gathered}
$$


which implies $\left(z_{1}^{m}, z_{2}^{m}\right) \longrightarrow\left(z_{1}, z_{2}\right)$ almost everywhere in A.
By multiplying (31) and (36) by $\theta(t) \in \mathscr{D}(0, T)$ and by integrating over $(0, T)$, it follows that

$$
\left\{\begin{array}{l}
-\frac{1}{l+1} \int_{0}^{T}\left(\left|u_{t}^{k}(t)\right|^{l} u_{t}^{k}(t), w^{j}\right) \theta^{\prime}(t) \mathrm{d} t+\int_{0}^{T} M\left(\left\|\nabla u^{k}(t)\right\|^{2}\right)\left(\nabla u^{k}(t), \nabla w^{j}\right) \theta(t) \mathrm{d} t+\int_{0}^{T}\left(\nabla u_{t t}^{k}, \nabla w^{j}\right) \theta(t) \mathrm{d} t \\
\left.-\int_{0}^{T} \int_{0}^{t} h_{1}(t-s)\left(\nabla u^{k}(s), \nabla w^{j}\right) \theta(t) \mathrm{d} s \mathrm{~d} t+\mu_{1} \int_{0}^{T}\left(\nabla z_{1}^{k}(., 1)\right), \nabla w^{j} \theta(t) \mathrm{d} t\right)=0, \\
-\frac{1}{l+1} \int_{0}^{T}\left(\left|v_{t}^{k}(t)\right|^{l} v_{t}^{k}(t), w^{j}\right) \theta r(t) \mathrm{d} t+\int_{0}^{T} M\left(\left\|\nabla v^{k}(t)\right\|^{2}\right)\left(\nabla v^{k}(t), \nabla w^{j}\right) \theta(t) \mathrm{d} t+\int_{0}^{T}\left(\nabla v_{t t}^{k}, \nabla w^{j}\right) \theta(t) \mathrm{d} t \\
\left.-\int_{0}^{T} \int_{0}^{t} h_{2}(t-s)\left(\nabla v^{k}(s), \nabla w^{j}\right) \theta(t) \mathrm{d} s \mathrm{~d} t+\mu_{2} \int_{0}^{T}\left(\nabla z_{2}^{k}(., 1)\right), \nabla w^{j} \theta(t) \mathrm{d} t\right)=0, \\
\int_{0}^{T} \int_{0}^{1} \int_{\Omega}\left(\tau(t) \frac{\partial}{\partial t} z_{1}^{k}+\left(1-\rho \tau^{\prime}(t)\right) \frac{\partial}{\partial \rho} z_{1}^{k}\right) \phi^{j} \theta(t) \mathrm{d} x \mathrm{~d} \rho \mathrm{d} t=0, \\
\int_{0}^{T} \int_{0}^{1} \int_{\Omega}\left(\tau(t) \frac{\partial}{\partial t} z_{2}^{k}+\left(1-\rho \tau^{\prime}(t)\right) \frac{\partial}{\partial \rho} z_{2}^{k}\right) \phi^{j} \theta(t) \mathrm{d} x \mathrm{~d} \rho \mathrm{d} t=0
\end{array}\right.
$$

for all $j=1 \ldots k$.

The convergence condition in (58)-(62), (68) and (67) are sufficient, thus we can pass to the limit in (70). Then, we have

$$
\left\{\begin{array}{l}
\int_{0}^{T} \int_{\Omega}\left(\left|u_{t}\right|^{l} u_{t t}-M\left(\|\nabla u(t)\|^{2}\right) \Delta u-\Delta u_{t t}+\int_{0}^{t} h_{1}(t-s) \Delta u(s) \mathrm{d} s-\mu_{1} \Delta z_{1}(., 1)\right) w^{j} \theta(t) \mathrm{d} x \mathrm{~d} t=0, \\
\int_{0}^{T} \int_{\Omega}\left(\left|v_{t}\right|^{l} v_{t t}-M\left(\|\nabla v(t)\|^{2}\right) \Delta v-\Delta v_{t t}+\int_{0}^{t} h_{2}(t-s) \Delta u(s) \mathrm{d} s-\mu_{2} \Delta z_{2}(., 1)\right) w^{j} \theta(t) \mathrm{d} x \mathrm{~d} t=0, \\
\int_{0}^{T} \int_{0}^{1} \int_{\Omega}\left(\tau(t) \frac{\partial}{\partial t} z_{1}+\left(1-\rho \tau^{\prime}(t)\right) \frac{\partial}{\partial \rho} z_{1}\right) \phi^{j} \theta(t) \mathrm{d} x \mathrm{~d} \rho \mathrm{d} t=0, \\
\int_{0}^{T} \int_{0}^{1} \int_{\Omega}\left(\tau(t) \frac{\partial}{\partial t} z_{2}+\left(1-\rho \tau^{\prime}(t)\right) \frac{\partial}{\partial \rho} z_{2}\right) \phi^{j} \theta(t) \mathrm{d} x \mathrm{~d} \rho \mathrm{d} t=0,
\end{array}\right.
$$

for all $j=1 \ldots k$. This completes the proof of Theorem 1 .

$$
I(t)=\tau(t) \int_{0}^{1} e^{-2 \tau(t) \rho}\left(\left\|\nabla z_{1}(x, \rho, t)\right\|^{2}+\left\|\nabla z_{1}(x, \rho, t)\right\|^{2}\right) \mathrm{d} \rho
$$

\section{Uniform Decay of the Energy (Proof of \\ Theorem 2)}

In this section we study the solution's asymptotic behavior of system (3).

To prove our main result, we construct a Lyapunov functional equivalent to $E$. For this, we define some functionals which allow us to obtain the desired estimate.

satisfies the estimate

(i) $|I(t)| \leq(1 / \xi) E(t)$.

(ii) $I^{\prime}(t) \leq-2 \tau(t) e^{-2 \tau_{1}} \int_{0}^{1}\left(\left\|\nabla z_{1}(x, \rho, t)\right\|^{2}+\| \nabla z_{2}(x, \rho\right.$, $\left.t) \|^{2}\right) d \rho-(1-d) e^{-2 \tau_{1}}\left(\left\|\nabla z_{1}(x, 1, t)\right\|^{2}+\| \nabla z_{2}(x, 1\right.$, $\left.t) \|^{2}\right)+\left\|\nabla u_{t}(x, t)\right\|^{2}+\left\|\nabla v_{t}(x, t)\right\|^{2}$.

Lemma 4. Let $\left(u, v, z_{1}, z_{2}\right)$ be a solution of problem (7). Then, the functional

Proof. (ii) Differentiating (72) with respect to $t$ and using (5)-(6) and Assumption 3, we get 


$$
\begin{aligned}
\frac{\mathrm{d}}{\mathrm{d} t} I(t)= & \tau^{\prime}(t) \int_{0}^{1} e^{-2 \tau(t) \rho}\left(\left\|\nabla z_{1}(x, \rho, t)\right\|^{2}+\left\|\nabla z_{2}(x, \rho, t)\right\|^{2}\right) \mathrm{d} \rho \\
& +\tau(t) \int_{\Omega} \int_{0}^{1}\left[e^{-2 \tau(t) \rho} \frac{\partial\left(\left|\nabla z_{1}(x, \rho, t)\right|^{2}+\left|\nabla z_{2}(x, \rho, t)\right|^{2}\right)}{\partial t}\right. \\
& \left.-2 \tau^{\prime}(t) \rho e^{-2 \tau(t) \rho}\left(\left|\nabla z_{1}(x, \rho, t)\right|^{2}+\left|\nabla z_{2}(x, \rho, t)\right|^{2}\right)\right] \mathrm{d} \rho \mathrm{d} x \\
= & \int_{\Omega} \int_{0}^{1} e^{-2 \tau(t) \rho}\left[\tau^{\prime}(t)\left(\left|\nabla z_{1}(x, \rho, t)\right|^{2}+\left|\nabla z_{2}(x, \rho, t)\right|^{2}\right)+\tau(t) \frac{\partial\left(\left|\nabla z_{1}(x, \rho, t)\right|^{2}+\left|\nabla z_{2}(x, \rho, t)\right|^{2}\right)}{\partial t}\right] \mathrm{d} \rho \mathrm{d} x \\
& -2 \int_{\Omega} \int_{0}^{1} \tau(t) \tau^{\prime}(t) \rho e^{-2 \tau(t) \rho}\left(\left\|\nabla z_{1}(x, \rho, t)\right\|^{2}+\left\|\nabla z_{2}(x, \rho, t)\right\|^{2}\right) \mathrm{d} \rho \mathrm{d} x \\
= & -\int_{0}^{1} e^{-2 \tau(t) \rho} \frac{\partial}{\partial \rho}\left(\left(1-\rho \tau^{\prime}(t)\right)\left(\left\|\nabla z_{1}(x, \rho, t)\right\|^{2}+\left\|\nabla z_{2}(x, \rho, t)\right\|^{2}\right)\right) \mathrm{d} \rho \\
& -2 \int_{0}^{1} \tau(t) \tau^{\prime}(t) \rho e^{-2 \tau(t) \rho}\left(\left\|\nabla z_{1}(x, \rho, t)\right\|^{2}+\left\|\nabla z_{2}(x, \rho, t)\right\|^{2}\right) \mathrm{d} \rho \\
= & -\int_{0}^{1}\left[\frac{\partial}{\partial \rho}\left(e^{-2 \tau(t) \rho}\left(1-\tau^{\prime}(t) \rho\right)\left(\left\|\nabla z_{1}(x, \rho, t)\right\|^{2}+\left\|\nabla z_{2}(x, \rho, t)\right\|^{2}\right)\right)\right. \\
& -2 \tau(t) e^{-2 \tau(t) \rho}\left(1-\tau^{\prime}(t) \rho\right)\left(\left\|\nabla z_{1}(x, \rho, t)\right\|^{2}+\left\|\nabla z_{2}(x, \rho, t)\right\|^{2}\right) \mathrm{d} \rho \\
\leq & -2 I(t)+\left\|\nabla u_{t}(x, t)\right\|^{2}+\left\|\nabla v_{t}(x, t)\right\|^{2}-e^{-2 \tau(t) \rho}(1-d)\left(\left\|\nabla z_{1}(x, \rho, t)\right\|^{2}+\left\|\nabla z_{2}(x, \rho, t)\right\|^{2}\right) . \\
& -2 \tau(t) \tau^{\prime}(t) \int_{0}^{1} \rho e^{-2 \tau(t) \rho}\left(\left\|\nabla z_{1}(x, \rho, t)\right\|^{2}+\left\|\nabla z_{2}(x, \rho, t)\right\|^{2}\right) \mathrm{d} \rho \\
= & \left.\left\|\nabla z_{1}(x, \rho, t)\right\|^{2}+\left\|\nabla z_{2}(x, \rho, t)\right\|^{2}\right)-e^{-2 \tau(t) \rho}\left(1-\tau^{\prime}(t)\right)\left(\left\|\nabla z_{1}(x, \rho, t)\right\|^{2}+\left\|\nabla z_{2}(x, \rho, t)\right\|^{2}\right) \\
& 2 \tau(t) \int_{0}^{1}\left[\left(1-\tau^{\prime}(t) \rho\right)+\tau^{\prime}(t) \rho\right] e^{-2 \tau(t) \rho}\left(\left|\nabla z_{1}(x, \rho, t)\right|^{2}+\left|\nabla z_{2}(x, \rho, t)\right|^{2}\right) \mathrm{d} \rho \mathrm{d} x \\
&
\end{aligned}
$$

Since $e^{-2 \tau(t) \rho}$ is a decreasing function for $\rho \in[0,1]$ and $\tau(t) \in\left[\tau_{0}, \tau_{1}\right]$, we deduce

$$
I(t) \geq \tau(t) \int_{0}^{1} e^{-2 \tau_{1}}\left(\left\|\nabla z_{1}(x, \rho, t)\right\|^{2}+\left\|\nabla z_{2}(x, \rho, t)\right\|^{2}\right) \mathrm{d} \rho .
$$

Thus, our proof is completed.

Lemma 5. Let $\left(u, v, z_{1}, z_{2}\right)$ be a solution of problem (7). Then, the functional

$$
\begin{aligned}
\phi(t)= & \frac{1}{l+1} \int_{\Omega}\left(\left|u_{t}\right|^{l} u_{t} u+\left|v_{t}\right|^{l} v_{t} v\right) \mathrm{d} x \\
& +\int_{\Omega} \nabla u_{t} \nabla u \mathrm{~d} x+\int_{\Omega} \nabla v_{t} \nabla v \mathrm{~d} x,
\end{aligned}
$$

satisfies the estimate

(i) $|\phi(t)| \leq(1 /(l+2))\left(\left\|u_{t}\right\|_{l+2}^{l+2}+\left\|v_{t}\right\|_{l+2}^{l+2}\right)+\left(\left((l+1)^{-1} /\right.\right.$ $\left.(l+2)) c_{s}^{l+2}+(c / 2)\right)\left(\|\nabla u\|^{l+2}+\|\nabla v\|^{l+2}\right)+(1 / 2)\left(\|\nabla u\|^{2}\right.$ $\left.+\|\nabla v\|^{2}\right)$.

(ii) $\phi^{\prime}(t) \leq(1 /(l+1))\left(\left\|u_{t}\right\|_{l+2}^{l+2}+\left\|v_{t}\right\|_{l+2}^{l+2}\right)+(\eta(a-k+$

$$
\begin{aligned}
& \left.1)+\alpha C_{s}-k\right)\left(\|\nabla u\|^{2}+\|\nabla v\|^{2}\right)+(1 / 4 \eta)\left[\left(h_{1} o \nabla u\right)(t)\right. \\
& \left.+\left(h_{1} o \nabla u\right)(t)\right]+\left(\mu_{1}^{2} / 4 \eta\right)\left\|\nabla z_{1}(x, 1, t)\right\|^{2}+\left(\mu_{2}^{2} / 4 \eta\right) \\
& \left\|\nabla z_{2}(x, 1, t)\right\|^{2}+\left\|\nabla u_{t}\right\|^{2}+\left\|\nabla v_{t}\right\|^{2} .
\end{aligned}
$$

Proof

(i) From Young's inequality, the Sobolev embedding, and $L^{l+2} \longrightarrow L^{2}$, we deduce 


$$
\begin{aligned}
|\phi(t)| \leq & \frac{1}{l+2}\left\|u_{t}\right\|_{l+2}^{l+2}+\frac{(l+1)^{-1}}{l+2}\|u\|_{l+2}^{l+2}+\frac{1}{l+2}\left\|v_{t}\right\|_{l+2}^{l+2}+\frac{(l+1)^{-1}}{l+2}\|v\|_{l+2}^{l+2} \\
& +\frac{1}{2}\left\|\nabla u_{t}\right\|^{2}+\frac{1}{2}\|\nabla u\|^{2}+\frac{1}{2}\left\|\nabla v_{t}\right\|^{2}+\frac{1}{2}\|\nabla v\|^{2} \\
\leq & \frac{1}{l+2}\left\|u_{t}\right\|_{l+2}^{l+2}+\frac{(l+1)^{-1}}{l+2} c_{s}^{l+2}\|\nabla u\|^{l+2}+\frac{1}{l+2}\left\|v_{t}\right\|_{l+2}^{l+2}+\frac{(l+1)^{-1}}{l+2} c_{s}^{l+2}\|\nabla v\|^{l+2} \\
& +\frac{1}{2}\left\|\nabla u_{t}\right\|^{2}+\frac{1}{2}\|\nabla u\|^{2}+\frac{1}{2}\left\|\nabla v_{t}\right\|^{2}+\frac{1}{2}\|\nabla v\|^{2} \\
\leq & \frac{1}{l+2}\left(\left\|u_{t}\right\|_{l+2}^{l+2}+\left\|v_{t}\right\|_{l+2}^{l+2}\right)+\left(\frac{(l+1)^{-1}}{l+2} c_{s}^{l+2}+\frac{c}{2}\right)\left(\|\nabla u\|^{l+2}+\|\nabla v\|^{l+2}\right)+\frac{1}{2}\left(\left\|\nabla u_{t}\right\|^{2}+\left\|\nabla v_{t}\right\|^{2}\right) .
\end{aligned}
$$

(i) Differentiating $\phi(t)$ with respect to $t$ and using the first and second equations of (7), we get

$$
\begin{aligned}
\phi^{\prime}(t)= & \frac{1}{l+1} \int_{\Omega}\left(\left|u_{t}\right|^{l} u_{t}\right)^{\prime} u \mathrm{~d} x+\frac{1}{l+1} \int_{\Omega}\left|u_{t}\right|^{l+2} \mathrm{~d} x+\frac{1}{l+1} \int_{\Omega}\left(\left|v_{t}\right|^{l} v_{t}\right)^{\prime} \mathrm{v} \mathrm{d} x+\frac{1}{l+1} \int_{\Omega}\left|v_{t}\right|^{l+2} \mathrm{~d} x \\
& +\int_{\Omega} \nabla u_{t t} \nabla u \mathrm{~d} x+\int_{\Omega} \nabla u_{t} \nabla u_{t} \mathrm{~d} x+\int_{\Omega} \nabla v_{t t} \nabla v \mathrm{~d} x+\int_{\Omega} \nabla v_{t} \nabla v_{t} \mathrm{~d} x \\
= & \int_{\Omega}\left[\left|u_{t}\right|^{l} u_{t}\right] u \mathrm{~d} x+\frac{1}{l+1}\left\|u_{t}\right\|_{l+2}^{l+2}+\int_{\Omega}\left[\left|v_{t}\right|^{l} v_{t}\right] \mathrm{v} \mathrm{d} x+\frac{1}{l+1}\left\|v_{t}\right\|_{l+2}^{l+2} \\
& -\int_{\Omega} \nabla u_{t t} u \mathrm{~d} x+\left\|\nabla u_{t}\right\|^{2}-\int_{\Omega} \nabla v_{t t} \nabla u \mathrm{~d} x+\left\|\nabla v_{t}\right\|^{2} \\
= & \frac{1}{l+1}\left(\left\|u_{t}\right\|_{l+2}^{l+2}+\left\|u_{t}\right\|_{l+2}^{l+2}\right)+\int_{\Omega}\left[\left|u_{t}\right|^{l} u_{t}-\Delta u_{t t}\right] u \mathrm{~d} x+\int_{\Omega}\left[\left|v_{t}\right|^{l} v_{t}-\Delta v_{t t}\right] u \mathrm{~d} x+\left\|\nabla u_{t}\right\|^{2}+\left\|\nabla v_{t}\right\|^{2} \\
= & \frac{1}{l+1}\left(\left\|u_{t}\right\|_{l+2}^{l+2}+\left\|u_{t}\right\|_{l+2}^{l+2}\right)+\int_{\Omega}\left[-\alpha v+M\left(\|\nabla u\|^{2}\right) \Delta u-\int_{0}^{t} h_{1}(t-s) \Delta u(s) \mathrm{d} s+\mu_{1} \Delta z_{1}(x, 1, t)\right] u \mathrm{~d} x \\
& +\int_{\Omega}\left[-\alpha u+M\left(\|\nabla v\|^{2}\right) \Delta v-\int_{0}^{t} h_{1}(t-s) \Delta v(s) \mathrm{d} s+\mu_{2} \Delta z_{2}(x, 1, t)\right] u \mathrm{~d} x+\left\|\nabla u_{t}\right\|^{2}+\left\|\nabla v_{t}\right\|^{2} \\
& \left.\frac{1}{l+1}\left(\left\|u_{t}\right\|_{l+2}^{l+2}+\left\|u_{t}\right\|_{l+2}^{l+2}\right)-M\left(\left\|\nabla u_{t}\right\|^{2}\right)\left\|\nabla v_{t}\right\|^{2}+\int_{\Omega} \nabla u(t) \int_{0}^{t} h_{1}(t-s) \Delta u(s) \mathrm{d} s \mathrm{~d} x-\mu_{1}\right]_{\Omega} \nabla z_{1}(x, 1, t) \nabla u \mathrm{~d} x \\
& \left.-M\left(\|\nabla v\|^{2}\right)\|\nabla v\|^{2}+\int_{\Omega}^{t} \nabla v(t) \int_{0}^{t} h_{2}(t-s) \Delta v(s) \mathrm{d} s \mathrm{~d} x-\mu_{2} \int_{\Omega} \nabla z_{2}(x, 1, t) \nabla v \mathrm{~d} x+\left\|\nabla u_{t}\right\|^{2}+\left\|\nabla v_{t}\right\|^{2}-2 \alpha\right]_{\Omega} u v \mathrm{~d} x .
\end{aligned}
$$

As $M(r) \geq a$ and making use Young's inequality, we obtain

$$
\begin{aligned}
\phi^{\prime}(t) \leq & \frac{1}{l+1}\left(\left\|u_{t}\right\|_{l+2}^{l+2}+\left\|v_{t}\right\|_{l+2}^{l+2}\right)-a\|\nabla u\|^{2}+\int_{\Omega} \nabla u(t) \int_{0}^{t} h_{1}(t-s) \nabla u(s) \mathrm{d} s \mathrm{~d} x+\frac{\mu_{1}^{2}}{4 \eta}\left\|\nabla z_{1}(x, 1, t)\right\|^{2}+\eta\|\nabla u\|^{2} \\
& -a\|\nabla v\|^{2}+\int_{\Omega} \nabla v(t) \int_{0}^{t} h_{2}(t-s) \nabla v(s) \mathrm{d} s \mathrm{~d} x+\frac{\mu_{2}^{2}}{4 \eta}\left\|\nabla z_{2}(x, 1, t)\right\|^{2}+\eta\|\nabla v\|^{2}+\left\|\nabla u_{t}\right\|^{2}+\left\|\nabla v_{t}\right\|^{2}
\end{aligned}
$$


By use of Young's inequality, we can estimate the third term in the right side as follows:

$$
\begin{aligned}
\int_{\Omega} \nabla u(t) \int_{0}^{t} h_{1}(t-s) \nabla u(s) \mathrm{d} s \mathrm{~d} x & \leq \int_{0}^{t} h(t-s) \int_{\Omega}|\nabla u(t) \nabla u(s)-\nabla u(t)| \mathrm{d} x \mathrm{~d} s+\|\nabla u(t)\|^{2} \int_{0}^{t} h_{1}(t-s) \mathrm{d} s \\
\leq & \eta\|\nabla u(t)\|^{2} \int_{0}^{t} h_{1}(s) \mathrm{d} s+\frac{1}{4 \eta} \int_{0}^{t} h_{1}(t-s)\|\nabla u(s)-\nabla u(t)\|^{2} \mathrm{~d} s \\
& +\|\nabla u(t)\|^{2} \int_{0}^{t} h_{1}(s) \mathrm{d} s \\
\leq & (1+\eta)(a-k)\|\nabla u(t)\|^{2}+\frac{1}{4 \eta}\left(h_{1} o \nabla u\right)(t) .
\end{aligned}
$$

Similarly,

$$
\begin{aligned}
\int_{\Omega} \nabla v(t) \int_{0}^{t} h_{2}(t-s) \nabla v(s) \mathrm{d} s \mathrm{~d} x \leq & (1+\eta)(a-k)\|\nabla v(t)\|^{2}+\frac{1}{4 \eta}\left(h_{2} o \nabla v\right)(t), \\
& -2 \alpha \int_{\Omega} u v \mathrm{~d} x \leq \alpha C_{s}\left(\|\nabla u\|^{2}+\|\nabla v\|^{2}\right) .
\end{aligned}
$$

Thus, our proof is completed.

Lemma 6. Let $(u, z)$ be a solution of problem (7). Then, the functional

$$
\psi(t)=\int_{\Omega}\left(\Delta u_{t}-\frac{1}{l+1}\left|u_{t}\right|^{l} u_{t}\right) \int_{0}^{t} h_{1}(t-s)(u(t)-u(s)) \mathrm{d} s \mathrm{~d} x+\int_{\Omega}\left(\Delta v_{t}-\frac{1}{l+1}\left|v_{t}\right|^{l} v_{t}\right) \int_{0}^{t} h_{2}(t-s)(v(t)-v(s)) \mathrm{d} s \mathrm{~d} x
$$

satisfies the estimates

(i) $|\psi(t)| \leq(1 / 2)\left(\left\|\nabla u_{t}\right\|^{2}+\left\|\nabla v_{t}\right\|^{2}\right)+(1 / 2)(a-k)(1+$ $\left.\left((l+1)^{-1} /(l+2)\right)(a-k)^{l} c_{s}^{l+2}\right)\left[\left(h_{1} o \nabla u\right)(t)+\left(h_{2} o\right.\right.$ $\nabla v)(t)]+\left((l+1)^{-1} /(l+2)\right)(a-k)^{l+2} c_{s}^{l+2} 2^{2 l+1}(\| \nabla$ $\left.u\left\|^{2(l+1)}+\right\| \nabla v \|^{2(l+1)}\right)+(1 /(l+2))\left(\left\|u_{t}\right\|_{l+2}^{l+2}+\left\|v_{t}\right\|_{l+2}^{l+2}\right)$.

(ii) $\psi(t) \leq \delta\left[(a-k)+\left((l+1)^{-1} /(l+2)\right)\left(h_{1}(0)\right)^{l+2} c_{s}^{l+2}\right.$ $\left.2^{2(l+1)}\right] M\left(\|\nabla u\|^{2}\right)\|\nabla u\|^{2}+\left(\left(\alpha C_{s} / 2\right)+2 \delta(a-k)^{2}\right)$ $\|\nabla u\|^{2}+\left(\left(M\left(\|\nabla u\|^{2}\right) / 4 \delta\right)+\left(\left(\alpha C_{s} / 2\right)+2 \delta+(1 / 2 \delta)\right)\right.$ $(a-k))\left(h_{1} o \nabla v\right)(t)-\left(h_{1}(0) / 4 \delta\right)\left(1+\left((l+1)^{-1} /(l+\right.\right.$ 2)) $\left.\left(h_{1}(0)\right)^{l} c_{s}^{l+2}\right)\left(h_{1}^{\prime} o \nabla v\right)(t)+\left(\delta-\int_{0}^{t} h_{1}(s) d s\right) \| \nabla$ $u_{t}\left\|^{2}+\mu_{1}^{2} \delta\right\| \nabla z_{1}(x, 1, t) \|^{2}+(1 /(l+1))\left(1-\int_{0}^{t} h_{1}(s)\right.$ $d s)\left\|u_{t}\right\|_{l+2}^{l+2}+\delta\left[(a-k)+\left((l+1)^{-1} /(l+2)\right)\left(h_{2}\right.\right.$ $\left.(0))^{l+2} c_{s}^{l+2} 2^{2(l+1)}\right] M\left(\|\nabla v\|^{2}\right)\|\nabla v\|^{2}+\left(\left(\alpha C_{s} / 2\right)+2 \delta\right.$ $\left.(a-k)^{2}\right)\|\nabla v\|^{2}+\left(\left(M\left(\|\nabla u\|^{2}\right) / 4 \delta\right)+\left(\left(\alpha C_{s} / 2\right)+2 \delta+\right.\right.$
$(1 / 2 \delta))(a-k))\left(h_{2} o \nabla v\right)(t)-\left(h_{2}(0) / 4 \delta\right)(1+((l+$ $\left.\left.1)^{-1} /(l+2)\right)\left(h_{2}(0)\right)^{l} c_{s}^{l+2}\right)\left(h_{2}^{\prime} o \nabla v\right)(t)+\left(\delta-\int_{0}^{t} h_{2}(s)\right.$ $d s)\left\|\nabla v_{t}\right\|^{2}+\mu_{2}^{2} \delta\left\|\nabla z_{2}(x, 1, t)\right\|^{2}+(1 /(l+1))\left(1-\int_{0}^{t}\right.$ $\left.h_{2}(s) d s\right)\left\|v_{t}\right\|_{l+2}^{l+2}$,

where $\delta>0$ and $c_{s}$ is the Sobolev embedding constant.

Proof

(i) $\psi(t)=-\int_{\Omega} \nabla u_{t} \int_{0}^{t} h_{2}(t-s)(\nabla u(t)-\nabla u(s)) \mathrm{d} s \mathrm{~d} x-$ $\int_{\Omega}(1 /(l+1))\left|u_{t}\right|^{l} u_{t} \int_{0}^{t} h_{2}(t-s)(u(t)-u(s)) \mathrm{d} s \mathrm{~d} x-$ $\int_{\Omega} \nabla v_{t} \int_{0}^{t} h_{2}(t-s)(\nabla v(t)-\nabla v(s)) \mathrm{d} s \mathrm{~d} x-\int_{\Omega}(1 /(l+$ 1)) $\left|v_{t}\right|^{l} v_{t} \int_{0}^{t} h_{2}(t-s)(v(t)-v(s)) \mathrm{d} s \mathrm{~d} x$.

We use Young's inequality with the conjugate exponents $p=(l+2) /(l+1)$ and $q=l+2$, and the second term in the right hand side can be estimated as 
14

Complexity

$$
\begin{aligned}
\left.\left|-\int_{\Omega} \frac{1}{l+1}\right| u_{t}\right|^{l} u_{t} \int_{0}^{t} h_{1}(t-s)(u(t)-u(s)) \mathrm{d} s \mathrm{~d} x \mid & \leq \frac{1}{l+1}\left|\int_{\Omega}\left(\left|u_{t}\right|^{l} u_{t}\right)\left(\int_{0}^{t} h_{1}((t-s) u(t)-u(s)) d s\right) \mathrm{d} x\right| \\
& \leq \frac{1}{l+1}\left[\left.\left.\frac{1}{p} \int_{\Omega}|| u_{t}\right|^{l} u_{t}\right|^{P} \mathrm{~d} x+\frac{1}{q} \int_{\Omega}\left|\int_{0}^{t} h_{1}((t-s) u(t)-u(s)) d s\right|^{q} \mathrm{~d} x\right] \\
& \leq \frac{1}{l+1}\left[\left.\left.\frac{1}{p} \int_{\Omega}|| u_{t}\right|^{l+1}\right|^{P} \mathrm{~d} x+\frac{1}{q} \int_{\Omega} \int_{0}^{t}\left(h_{1}((t-s) u(t)-u(s)) d s\right)^{q} \mathrm{~d} x\right] \\
& \leq \frac{1}{l+2}\left\|u_{t}\right\|_{l+2}^{l+2}+\frac{(l+1)^{-1}}{l+2} \int_{\Omega}\left[\int_{0}^{t}\left(h_{1}(t-s)\right)^{\frac{l+1}{+2}}\left(\left(h_{1}(t-s)\right)^{\frac{1}{l+2}}|u(t)-u(s)|\right) \mathrm{d} s\right]^{l+2} \mathrm{~d} x
\end{aligned}
$$

We have by Hölder's inequality

$$
\begin{aligned}
& \int_{\Omega}\left[\int_{0}^{t}\left(h_{1}(t-s)\right)^{((l+1) /(l+2))}\left(\left(h_{1}(t-s)\right)^{(1 /(l+2))}|u(t)-u(s)|\right) \mathrm{d} s\right]^{l+2} \mathrm{~d} x \\
& \quad \leq \int_{\Omega}\left[\left(\int_{0}^{t}\left(\left(h_{1}(t-s)\right)^{((l+1) /(l+2))}\right)^{P} \mathrm{~d} s\right)^{1 / P}\left(\int_{0}^{t}\left(\left(h_{1}(t-s)\right)^{(1 /(l+2))}|u(t)-u(s)|\right)^{q} \mathrm{~d} s\right)^{1 / q}\right]^{l+2} \mathrm{~d} x \\
& \leq \int_{\Omega}\left(\int_{0}^{t} h_{1}(t-s) \mathrm{d} s\right)^{((l+1) /(l+2))}\left[\left(\int_{0}^{t} h_{1}(t-s) \mathrm{d} s|u(t)-u(s)|^{l+2} \mathrm{~d} s\right)^{1 / l+2}\right]^{l+2} \mathrm{~d} x \\
& \leq\left(\int_{0}^{t} h_{1}(t-s) \mathrm{d} s\right)^{l+1} \int_{0}^{t} h_{1}(t-s)\|u(t)-u(s)\|_{l+2}^{l+2} \mathrm{~d} s \\
& \leq(a-k)^{l+1} c_{s}^{l+2} \int_{0}^{t} \sqrt{h_{1}(t-s)} \sqrt{h_{1}(t-s)\|\nabla u(t)-\nabla u(s)\|^{l+1}\|\nabla u(t)-\nabla u(s)\| \mathrm{d} s} \\
& \leq(a-k)^{l+1} c_{s}^{l+2}\left(\frac{1}{2} \int_{0}^{t} h_{1}(t-s)\|\nabla u(t)-\nabla u(s)\|^{2 l+2} \mathrm{~d} s+\frac{1}{2} \int_{0}^{t} h_{1}(t-s)\|\nabla u(t)-\nabla u(s)\|^{2} \mathrm{~d} s\right) \\
& \leq(a-k)^{l+1} c_{s}^{l+2}\left(2^{2 l+1}(a-k)\|\nabla u(t)\|^{2(l+1)}+\frac{1}{2}\left(h_{1} o \nabla u\right)(t)\right) . \\
& \leq\left(a-k c_{s}^{l+2}\left(\frac{1}{2} \int_{0}^{t} h_{1}(t-s)\|2 \nabla u(t)\|^{2 l+2} \mathrm{~d} s+\frac{1}{2}\left(h_{1} o \nabla u\right)(t)\right)\right.
\end{aligned}
$$

Combining (83) with (82), we obtain

$$
\begin{aligned}
& \left.\left|-\int_{\Omega} \frac{1}{l+1}\right| u_{t}\right|^{l} u_{t} \int_{0}^{t} h_{1}(t-s)(u(t)-u(s)) \mathrm{d} s \mathrm{~d} x \mid \leq \frac{1}{l+2}\left\|u_{t}\right\|_{l+2}^{l+2} \\
& \quad+\frac{(l+1)^{-1}}{l+2}\left[(a-k)^{l+1} c_{s}^{l+2}\left(2^{2 l+1}(a-k)\|\nabla u(t)\|^{2(l+1)}+\frac{1}{2}\left(h_{1} o \nabla u\right)(t)\right)\right] .
\end{aligned}
$$


Similarly, we get

$$
\begin{aligned}
\left|-\int_{\Omega} \nabla u_{t} \int_{0}^{t} h_{1}(t-s)(\nabla u(t)-\nabla u(s)) \mathrm{d} s \mathrm{~d} x\right| & \leq \frac{1}{2}\left\|\nabla u_{t}\right\|^{2}+\frac{1}{2} \int_{\Omega}\left(\int_{0}^{t} h_{1}(t-s)|\nabla u(t)-\nabla u(s)| \mathrm{d} s\right)^{2} \mathrm{~d} x \\
& \leq \frac{1}{2}\left\|\nabla u_{t}\right\|^{2}+\frac{1}{2}(a-k)\left(h_{1} o \nabla u\right)(t) .
\end{aligned}
$$

Similarly,

$$
\left\{\begin{array}{l}
\left.\left|-\int_{\Omega} \frac{1}{l+1}\right| v_{t}\right|^{l} v_{t} \int_{0}^{t} h_{2}(t-s)(v(t)-v(s)) \mathrm{d} s \mathrm{~d} x \mid \leq \frac{1}{l+2}\left\|v_{t}\right\|^{l+2} \\
\quad+\frac{(l+1)^{-1}}{l+2}\left[(a-k)^{l+1} c_{s}^{l+2}\left(2^{2 l+1}(a-k)\|\nabla v(t)\|^{2(l+1)}+\frac{1}{2}\left(h_{2} o \nabla v\right)(t)\right)\right] \\
\left|-\int_{\Omega} \Omega \nabla v_{t} \int_{0}^{t} h_{2}(t-s)(\nabla v(t)-\nabla v(s)) \mathrm{d} s \mathrm{~d} x\right| \leq \frac{1}{2}\|\nabla v(t)\|^{2}+\frac{1}{2}(a-k)\left(h_{2} o \nabla v\right)(t)
\end{array}\right.
$$

Combining (84)-(86), we deduce (i).

(ii) Using the Leibniz formula and the first and second equations of (7), we have

$$
\begin{aligned}
\psi^{\prime}(t)= & \int_{\Omega}\left(\Delta u_{t t}-\left|u_{t}\right|^{l} u_{t t}\right) \int_{0}^{t} h_{1}(t-s)(u(t)-u(s)) \mathrm{d} s \mathrm{~d} x \\
& +\int_{\Omega}\left(\Delta u_{t}-\frac{1}{l+1}\left|u_{t}\right|^{l} u_{t}\right)\left(\int_{0}^{t}\left(h_{1}^{\prime}(t-s)(u(t)-u(s))\right)+h_{1}(t-s) u_{t}(t) \mathrm{d} s\right) \mathrm{d} x \\
& +\int_{\Omega}\left(\Delta v_{t t}-\left|v_{t}\right|^{l} v_{t t}\right) \int_{0}^{t} h_{2}(t-s)(v(t)-v(s)) \mathrm{d} s \mathrm{~d} x \\
& +\int_{\Omega}\left(\Delta v_{t}-\frac{1}{l+1}\left|v_{t}\right|^{l} v_{t}\right)\left(\int_{0}^{t}\left(h_{2}^{\prime}(t-s)(v(t)-v(s))\right)+h_{2}(t-s) v_{t}(t) \mathrm{d} s\right) \mathrm{d} x \\
= & -\alpha \int_{\Omega} v(t) \int_{0}^{t} h_{1}(t-s)(u(t)-u(s)) \mathrm{d} s \mathrm{~d} x+\int_{\Omega} M(\| \nabla u)^{2} \nabla u(t) \int_{0}^{t} h_{1}(t-s)(\nabla u(t)-\nabla u(s)) \mathrm{d} s \mathrm{~d} x \\
& -\int_{\Omega} \int_{0}^{t} h_{1}(t-s) \nabla u(s) \mathrm{d} s \int_{0}^{t} h_{1}(t-s)(\nabla u(t)-\nabla u(s)) \mathrm{d} s \mathrm{~d} x \\
& +\mu_{1} \int_{\Omega} \nabla z_{1}(x, 1, t) \int_{0}^{t} h_{1}(t-s)(\nabla u(t)-\nabla u(s)) \mathrm{d} s \mathrm{~d} x \\
& -\int_{\Omega} \nabla u_{t} \int_{0}^{t} h_{1}^{\prime}(t-s)(\nabla u(t)-\nabla u(s)) \mathrm{d} s \mathrm{~d} x-\frac{1}{l+1} \int_{\Omega}\left|u_{t}\right|^{l} u_{t}^{t} \int_{0}^{t} h_{1}^{\prime}(t-s)(\nabla u(t)-\nabla u(s)) \mathrm{d} s \mathrm{~d} x \\
& -\frac{1}{l+1}\left\|_{t}\right\|_{l+2}^{l+2} \int_{0}^{t} h_{1}(s) \mathrm{d} s
\end{aligned}
$$




$$
\begin{aligned}
& -\alpha \int_{\Omega} u(t) \int_{0}^{t} h_{2}(t-s)(v(t)-v(s)) \mathrm{d} s \mathrm{~d} x+\int_{\Omega} M(\|\nabla v\|)^{2} \nabla v(t) \int_{0}^{t} h_{2}(t-s)(\nabla v(t)-\nabla v(s)) \mathrm{d} s \mathrm{~d} x \\
& -\int_{\Omega} \int_{0}^{t} h_{2}(t-s) \nabla v(s) \mathrm{d} s \int_{0}^{t} h_{2}(t-s)(\nabla v(t)-\nabla v(s)) \mathrm{d} s \mathrm{~d} x \\
& +\mu_{2} \int_{\Omega} \nabla z_{2}(x, 1, t) \int_{0}^{t} h_{2}(t-s)(\nabla v(t)-\nabla v(s)) \mathrm{d} s \mathrm{~d} x \\
& -\int_{\Omega} \nabla v_{t} \int_{0}^{t} h_{2}^{\prime}(t-s)(\nabla v(t)-\nabla v(s)) \mathrm{d} s \mathrm{~d} x-\frac{1}{l+1} \int_{\Omega}\left|v_{t}\right|^{l} v_{t} \int_{0}^{t} h_{2}^{\prime}(t-s)(\nabla v(t)-\nabla v(s)) \mathrm{d} s \mathrm{~d} x \\
& -\left\|\nabla v_{t}\right\|^{2} \int_{0}^{t} h_{2}(s) \mathrm{d} s-\frac{1}{l+1}\left\|v_{t}\right\|_{l+2}^{l+2} \int_{0}^{t} h_{2}(s) \mathrm{d} s \\
& =I_{1}+I_{2}+I_{3}+I_{4}+I_{5}+I_{6}-\left\|\nabla u_{t}\right\|^{2} \int_{0}^{t} h_{1}(s) \mathrm{d} s-\frac{1}{l+1}\left\|u_{t}\right\|^{l+2} \int_{0}^{t} h_{1}(s) \mathrm{d} s \\
& -\left\|\nabla v_{t}\right\|^{2} \int_{0}^{t} h_{2}(s) \mathrm{d} s-\frac{1}{l+1}\left\|v_{t}\right\|^{l+2} \int_{0}^{t} h_{2}(s) \mathrm{d} s,
\end{aligned}
$$

where

$$
\left\{\begin{array}{l}
I_{1}=\int_{\Omega} M\left(\|\nabla u\|^{2}\right) \nabla u(t) \int_{0}^{t} h_{1}(t-s)(\nabla u(t)-\nabla u(s)) \mathrm{d} s \mathrm{~d} x+\int_{\Omega} M\left(\|\nabla v\|^{2}\right) \nabla v(t) \int_{0}^{t} h_{2}(t-s)(\nabla v(t)-\nabla v(s)) \mathrm{d} s \mathrm{~d} x \\
I_{2}=-\int_{\Omega} \int_{0}^{t} h_{1}(t-s) \nabla u(s) \mathrm{d} s \int_{0}^{t} h_{1}(t-s)(\nabla u(t)-\nabla u(s)) \mathrm{d} s \mathrm{~d} x \\
\quad-\int_{\Omega} \int_{0}^{t} h_{2}(t-s) \nabla v(s) \mathrm{d} s \int_{0}^{t} h_{2}(t-s)(\nabla v(t)-\nabla v(s)) \mathrm{d} s \mathrm{~d} x \\
I_{3}=\mu_{1} \int_{\Omega} \nabla z_{1}(x, 1, t) \int_{0}^{t} h_{1}(t-s)(\nabla u(t)-\nabla u(s)) \mathrm{d} s \mathrm{~d} x+\mu_{2} \int_{\Omega} \nabla z_{2}(x, 1, t) \int_{0}^{t} h_{2}(t-s)(\nabla v(t)-\nabla v(s)) \mathrm{d} s \mathrm{~d} x \\
I_{4}=-\int_{\Omega} \nabla u_{t} \int_{0}^{t} h_{1}^{\prime}(t-s)(\nabla u(t)-\nabla u(s)) \mathrm{d} s \mathrm{~d} x-\int_{\Omega} \nabla v_{t} \int_{0}^{t} h_{2}^{\prime}(t-s)(\nabla v(t)-\nabla v(s)) \mathrm{d} s \mathrm{~d} x \\
I_{5}=-\frac{1}{l+1} \int_{\Omega}\left|u_{t}\right|^{l} u_{t} \int_{0}^{t} h_{1}^{\prime}(t-s)(\nabla u(t)-\nabla u(s)) \mathrm{d} s \mathrm{~d} x-\frac{1}{l+1} \int_{\Omega}\left|v_{t}\right|^{l} v_{t} \int_{0}^{t} h_{2}^{\prime}(t-s)(\nabla v(t)-\nabla v(s)) \mathrm{d} s \mathrm{~d} x \\
I_{6}=-\alpha \int_{\Omega} v(t) \int_{0}^{t} h_{1}^{\prime}(t-s)(\nabla u(t)-\nabla u(s)) \mathrm{d} s \mathrm{~d} x-\alpha \int_{\Omega} u(t) \int_{0}^{t} h_{2}^{\prime}(t-s)(\nabla v(t)-\nabla v(s)) \mathrm{d} s \mathrm{~d} x .
\end{array}\right.
$$

In what follows we will estimate $I_{1}, \ldots, I_{6}$.

For $I_{1}$, we use Hölder and Young's inequalities with $p=q=2$, and we get 


$$
\begin{aligned}
\left|I_{1}\right| \leq & M\left(\|\nabla u\|^{2}\right) \int_{\Omega}|\nabla u(t)|\left(\int_{0}^{t} h_{1}(s) \mathrm{d} s\right)^{1 / 2}\left(\int_{0}^{t} h_{1}(s) \mid \nabla u(t)-\nabla u\left(\left.(s)\right|^{2} \mathrm{~d} s\right)^{1 / 2} \mathrm{~d} x\right. \\
& +M\left(\|\nabla v\|^{2}\right) \int_{\Omega}|\nabla v(t)|\left(\int_{0}^{t} h_{2}(s) \mathrm{d} s\right)^{1 / 2}\left(\int_{0}^{t} h_{2}(t-s)|\nabla v(t)-\nabla v(s)|^{2} \mathrm{~d} s\right)^{1 / 2} \mathrm{~d} x \\
\leq & M\left(\|\nabla u\|^{2}\right)\left[\delta \int_{\Omega}|\nabla u(t)|^{2}\left(\int_{0}^{t} h_{1}(s) \mathrm{d} s\right) \mathrm{d} x+\frac{1}{4 \delta} \int_{\Omega} \int_{0}^{t} h_{1}(t-s)|\nabla u(t)-\nabla u(s)|^{2} \mathrm{~d} s \mathrm{~d} x\right] \\
& +M\left(\|\nabla v\|^{2}\right)\left[\delta \int_{\Omega}|\nabla v(t)|^{2}\left(\int_{0}^{t} h_{2}(s) \mathrm{d} s\right) \mathrm{d} x+\frac{1}{4 \delta} \int_{\Omega} \int_{0}^{t} h_{2}(t-s)|\nabla v(t)-\nabla v(s)|^{2} \mathrm{~d} s \mathrm{~d} x\right] \\
\leq & M\left(\|\nabla u\|^{2}\right)\left(\delta(a-k)\|\nabla u(t)\|^{2}+\frac{1}{4 \delta}\left(h_{1} o \nabla u\right)(t)\right)+M\left(\|\nabla v\|^{2}\right)\left(\delta(a-k)\|\nabla v(t)\|^{2}+\frac{1}{4 \delta}\left(h_{1} o \nabla v\right)(t)\right) .
\end{aligned}
$$

Similarly,

$$
\begin{aligned}
&\left|I_{2}\right| \leq \delta \int_{\Omega}\left(\int_{0}^{t} h_{1}(t-s)|\nabla u(s)| \mathrm{d} s\right)^{2} \mathrm{~d} x+\frac{1}{4 \delta} \int_{\Omega}\left(\int_{0}^{t} h_{1}(t-s)|\nabla u(t)-\nabla u(s)| \mathrm{d} s\right)^{2} \mathrm{~d} x \\
&+\delta \int_{\Omega}\left(\int_{0}^{t} h_{2}(t-s)|\nabla v(s)| \mathrm{d} s\right)^{2} \mathrm{~d} x+\frac{1}{4 \delta} \int_{\Omega}\left(\int_{0}^{t} h_{2}(t-s)|\nabla v(t)-\nabla v(s)| \mathrm{d} s\right)^{2} \mathrm{~d} x \\
& \leq \delta \int_{\Omega}\left(\int_{0}^{t} h_{1}(t-s)(|\nabla u(s)-\nabla u(t)|+|\nabla u(t)|) \mathrm{d} s\right)^{2} \mathrm{~d} x+\frac{1}{4 \delta}\left(\int_{0}^{t} h_{1}(s) \mathrm{d} s\left(h_{1} o \nabla u\right)(t)\right) \\
&+\delta \int_{\Omega}\left(\int_{0}^{t} h_{2}(t-s)(|\nabla v(s)-\nabla v(t)|+|\nabla v(t)|) \mathrm{d} s\right)^{2} \mathrm{~d} x+\frac{1}{4 \delta}\left(\int_{0}^{t} h_{2}(s) \mathrm{d} s\left(h_{2} o \nabla v\right)(t)\right) \\
& \leq 2 \delta\|\nabla u(t)\|^{2}\left(\int_{0}^{t} h_{1}(t) \mathrm{d} s^{2} \mathrm{~d} x+\left(2 \delta+\frac{1}{4 \delta}\right)\right) \int_{0}^{t} h_{1}((s) \mathrm{d} s)\left(h_{1} o \nabla u\right)(t) \\
&+2 \delta\|\nabla v(t)\|^{2}\left(\int_{0}^{t} h_{2}(t) \mathrm{d} s^{2} \mathrm{~d} x+\left(2 \delta+\frac{1}{4 \delta}\right)\right) \int_{0}^{t} h_{2}((s) \mathrm{d} s)\left(h_{1} o \nabla v\right)(t) \\
& \leq 2 \delta\|\nabla u(t)\|^{2}(a-k)^{2}+\left(2 \delta+\frac{1}{4 \delta}\right)(a-k)\left(h_{1} o \nabla u\right)(t)+2 \delta\|\nabla v(t)\|^{2}(a-k)^{2}+\left(2 \delta+\frac{1}{4 \delta}\right)(a-k)\left(h_{2} o \nabla v\right)(t) \\
&\left|I_{3}\right| \leq \delta\left(\mu_{1}^{2}\left\|\nabla z_{1}(x, 1, t)\right\|^{2}+\mu_{2}^{2}\left\|\nabla z_{2}(x, 1, t)\right\|^{2}\right)+\frac{(a-k)}{4 \delta}\left(h_{1} o \nabla u\right)(t)+\frac{(a-k)}{4 \delta}\left(h_{2} o \nabla v\right)(t) \\
&+\delta\left\|\nabla u_{t}\right\|^{2}-\frac{h_{1}(0)}{4 \delta}\left(h_{1}^{\prime} o \nabla u\right)(t)+\delta\left\|\nabla v_{t}\right\|^{2}-\frac{h_{2}(0)}{4 \delta}\left(h_{2}^{\prime} o \nabla u\right)(t) \\
&\left|I_{4}\right| \leq \delta \int_{\Omega}\left|\nabla u_{t}\right|^{2} \mathrm{~d} x+\frac{1}{4 \delta} \int_{\Omega}\left(\int_{0}^{t}\left|h_{1}^{\prime}(t-s)\right||\nabla u(t)-\nabla u(s)| \mathrm{d} s\right)^{2} \mathrm{~d} x \\
&+\delta \int_{\Omega}\left|\nabla v_{t}\right|^{2} \mathrm{~d} x+\frac{1}{4 \delta} \int_{\Omega}\left(\int_{0}^{t}\left|h_{2}^{\prime}(t-s)\right||\nabla v(t)-\nabla v(s)| \mathrm{d} s\right)^{2} \mathrm{~d} x \\
& \leq \delta\left\|\nabla u_{t}\right\|^{2}+\frac{1}{4 \delta} \int_{0}^{t}\left(-h_{1}^{\prime}(t-s)\right) \mathrm{d} s \int_{\Omega} \int_{0}^{t}\left(-h_{1}^{\prime}(t-s)\right)|\nabla u(t)-\nabla u(s)|^{2} \mathrm{~d} s \mathrm{~d} x \\
&+\delta\left\|\nabla v_{t}\right\|^{2}+\frac{1}{4 \delta} \int_{0}^{t}\left(-h_{2}^{\prime}(t-s)\right) \mathrm{d} s \int_{\Omega}^{t}\left(-h_{2}^{\prime}(t-s)\right)|\nabla v(t)-\nabla v(s)|^{2} \mathrm{~d} s \mathrm{~d} x
\end{aligned}
$$

and since $l \leq \gamma$, we follow almost the same steps to obtain $|\psi(t)|)$ 


$$
\begin{aligned}
& \left|I_{5}\right| \leq \frac{1}{l+2}\left(\left\|u_{t}\right\|_{l+2}^{l+2}+\left\|v_{t}\right\|_{l+2}^{l+2}\right)+\frac{(l+1)^{-1}}{l+2}\left[\left(h_{1}(0)\right)^{l+1} \int_{0}^{t}\left(-h_{1}^{\prime}(t-s)\right)\|u(t)-u(s)\|_{l+2}^{l+2} \mathrm{~d} s\right. \\
& +\left(h_{2}(0)\right)^{l+1} \int_{0}^{t}\left(-h_{2}^{\prime}(t-s)\right)\|v(t)-v(s)\|_{l+2}^{l+2} \mathrm{~d} s \\
& \leq \frac{1}{l+2}\left(\left\|u_{t}\right\|_{l+2}^{l+2}+\left\|v_{t}\right\|_{l+2}^{l+2}\right)+\frac{(l+1)^{-1}}{l+2} c_{s}^{l+2}\left[\left(h_{1}(0)\right)^{l+1} \int_{0}^{t}\left(-h_{1}^{\prime}(t-s)\right)\|\nabla u(t)-\nabla u(s)\|^{1+2} \mathrm{~d} s\right. \\
& +\left(h_{2}(0)\right)^{l+1} \int_{0}^{t}\left(-h_{2}^{\prime}(t-s)\right)\|\nabla u(t)-\nabla u(s)\|^{1+2} \mathrm{~d} s \\
& \leq \frac{1}{l+2}\left(\left\|u_{t}\right\|_{l+2}^{l+2}+\left\|v_{t}\right\|_{l+2}^{l+2}\right)+\frac{(l+1)^{-1}}{l+2} c_{s}^{l+2}\left(h_{1}(0)\right)^{l+1}\left[\delta 2^{2(l+1)} h_{1}(0)\|\nabla u(t)\|^{2(l+1)}-\frac{1}{4 \delta}\left(h_{1}^{\prime} o \nabla u\right)(t)\right] \\
& +\frac{(l+1)^{-1}}{l+2} c_{s}^{l+2}\left(h_{2}(0)\right)^{l+1}\left[\delta 2^{2(l+1)} h_{2}(0)\|\nabla v(t)\|^{2(l+1)}-\frac{1}{4 \delta}\left(h_{2}^{\prime} o \nabla v\right)(t)\right] \\
& \leq \frac{1}{l+2}\left(\left\|u_{t}\right\|_{l+2}^{l+2}+\left\|v_{t}\right\|_{l+2}^{l+2}\right)+\frac{(l+1)^{-1}}{l+2} c_{s}^{l+2}\left(h_{1}(0)\right)^{l+1}\left[\delta 2^{2(l+1)} h_{1}(0) M\left(\|\nabla u(t)\|^{2}\right)\|\nabla u(t)\|^{2}-\frac{1}{4 \delta}\left(h_{1}^{\prime} o \nabla u\right)(t)\right] \\
& +\frac{(l+1)^{-1}}{l+2} c_{s}^{l+2}\left(h_{2}(0)\right)^{l+1}\left[\delta 2^{2(l+1)} h_{2}(0) M\left(\|\nabla v(t)\|^{2}\right)\|\nabla v(t)\|^{2}-\frac{1}{4 \delta}\left(h_{2}^{\prime} o \nabla v\right)(t)\right] \\
& \left|I_{6}\right| \leq \frac{\alpha C_{s}}{2}\left(\|\nabla u(t)\|^{2}+\|\nabla v(t)\|^{2}+(a-k)\left[\left(h_{1} o \nabla u\right)(t)+\left(h_{2} o \nabla v\right)(t)\right]\right) .
\end{aligned}
$$

Combining (87) and (89)-(94), we finish the proof.

Proof of Theorem 1.2. Now, for $M, \varepsilon_{1}>0$, we introduce the following functional:

$$
F(t)=M E(t)+\varepsilon_{1} \phi(t)+\psi(t)+I(t) \sim E(t) .
$$

Indeed, to prove $F(t) \sim E(t)$, we show that there exist two positive constants $\kappa_{1}$ and $\kappa_{2}$ such that

$$
\kappa_{1} E(t) \leq F(t) \leq \kappa_{2} E(t) .
$$

From (i) of Lemmas 4-6 and recalling the fact that $l \leq \gamma$, we get

$$
\begin{aligned}
\left|\varepsilon_{1} \phi(t)+\psi(t)+I(t)\right| \leq & \frac{\varepsilon_{1}+1}{l+2}\left(\left\|u_{t}\right\|_{l+2}^{l+2}+\left\|v_{t}\right\|_{l+2}^{l+2}\right)+\frac{\varepsilon_{1}+1}{2}\left(\left\|\nabla u_{t}\right\|^{2}\right)+\left(\left\|\nabla v_{t}\right\|^{2}\right) \\
& +\left(\frac{\varepsilon_{1} c}{2}+\frac{(l+1)^{-1}}{(l+2)} c_{s}^{l+2}\left(\varepsilon_{1}+2^{2 l+1}(a-k)^{l+2}\right)\right)\left(\|\nabla u\|^{2(\gamma+1)}+\|\nabla v\|^{2(\gamma+1)}\right) \\
& +\frac{a-k}{2}\left(1+\frac{(l+1)^{-1}}{(l+2)}(a-k)^{l} c_{s}^{l+2}\right)\left(\left(h_{1} o \nabla u\right)(t)+\left(h_{2} o \nabla v\right)(t)\right)+\frac{1}{\xi} E(t) \\
\leq & \kappa E(t),
\end{aligned}
$$

where $\kappa>0$ depending on $\varepsilon_{1}, a, b, l, c, c_{s}, k, \xi$. If we choose $M=\kappa+\epsilon$, we can obtain our result.
By recalling Lemmas 3-6 and by Assumption 2, we deduce that for $t \geq t_{0}>0$, 


$$
\begin{aligned}
F^{\prime}(t)= & M E^{\prime}(t)+\varepsilon_{1} \phi^{\prime}(t)+\psi^{\prime}(t)+I^{\prime}(t) \\
\leq & \left(\mu^{2} \delta+\varepsilon_{1} \frac{\mu^{2}}{4 \eta}-(1-d) e^{-2 \tau_{1}}-M \beta\right)\left(\left\|\nabla z_{1}(x, 1, t)\right\|^{2}+\left\|\nabla z_{2}(x, 1, t)\right\|^{2}\right) \\
& -2 \tau(t) e^{-2 \tau_{1}} \int_{0}^{1}\left(\left\|\nabla z_{1}(x, p, t)\right\|^{2}+\left\|\nabla z_{2}(x, p, t)\right\|^{2}\right) \mathrm{d} \rho \\
& -\left(\varepsilon_{1}[k-\eta(a-k+1)]-\frac{\alpha C_{S}}{2}-2 \delta(a-k)^{2}-\delta\left[(a-k)+\frac{(l+1)^{-1}}{(l+2)}\left(h_{2} c_{s}\right)^{l+2} 2^{2(l+1)}\right] M_{0}\right)\left(\|\nabla u\|^{2}+\|\nabla v\|^{2}\right) \\
& +\left[\left(\frac{M}{2}-\frac{h_{1}}{4 \delta}\left(1+\frac{(l+1)^{-1}}{(l+2)} h_{1}^{l} c_{s}^{l+2}\right)-\frac{1}{\zeta}\left(\frac{\varepsilon_{1}}{4 \eta}+\frac{M_{0}}{4 \delta}+\left(\frac{\alpha C_{S}}{2}+2 \delta+\frac{1}{2 \delta}\right)(a-k)\right)\right]\left(h_{1}^{\prime} o \nabla u\right)(t)+\left(h_{2}^{\prime} o \nabla u\right)(t)\right. \\
& -\frac{1}{l+1}\left(h_{0}-1-\varepsilon_{1}\right)\left(\left\|u_{t}\right\|_{l+2}^{l+2}+\left\|v_{t}\right\|_{l+2}^{l+2}\right)-\left(h_{0}-\delta-M \lambda-1-\varepsilon_{1}\right)\left(\left\|\nabla u_{t}\right\|^{2}+\left\|\nabla v_{t}\right\|^{2}\right)
\end{aligned}
$$

where $\quad M_{0}=\max \left\{M\left(\|\nabla u\|^{2}\right), M\left(\|\nabla v\|^{2}\right)\right\}, \quad h_{0}=\min$ $\left\{\int_{0}^{t_{0}} h_{1}(s) \mathrm{d} s, \int_{0}^{t_{0}} h_{2}(s) \mathrm{d} s\right\}, \quad h_{1}=\min \left\{h_{1}(0), h_{2}(0)\right\}, \quad h_{2}=\min$ $\left\{h_{1}(0), h_{2}(0)\right\}$, and $\zeta=\max \left\{\zeta_{1}, \zeta_{1}\right\}$.

Let $\epsilon>0$ be sufficiently small so $M$ is fixed; we take $h_{0}-$ $M \lambda-1>\varepsilon_{1}$ and $\delta$ small enough such that

$$
\begin{aligned}
& a_{3}=h_{0}-1-\varepsilon_{1}>0, \\
& a_{4}=h_{0}-\delta-M \lambda-1-\varepsilon_{1} .
\end{aligned}
$$

$$
\begin{aligned}
& a_{1}=\mu_{1}^{2} \delta+\varepsilon_{1} \frac{\mu^{2}}{4 \eta}-(1-d) e^{-2 \tau_{1}}-M \beta>0 \\
& a_{2}=\varepsilon_{1}[k-\eta[a-k+1]]-\frac{\alpha C_{S}}{2}-2 \delta(a-k)^{2}-\delta\left((a-k)+\frac{(l+1)^{-1}}{(l+2)}\left(h_{2} c_{s}\right)^{l+2} 2^{2(l+1)}\right) M_{0}>0 \\
& a_{5}=\frac{M}{2}-\frac{h_{1}}{4 \delta}\left(1+\frac{(l+1)^{-1}}{(l+2)} h_{1}^{l} c_{s}^{l+2}\right)-\frac{1}{\zeta}\left(\frac{\varepsilon_{1}}{4 \eta}+\frac{M_{0}}{4 \delta}+\left(\frac{\alpha C_{S}}{2}+2 \delta+\frac{1}{2 \delta}\right)(a-k)\right)<0 .
\end{aligned}
$$

Thus,

$$
\begin{aligned}
F^{\prime}(t) \leq & -a_{3} \frac{1}{l+2}\left(\left\|u_{t}\right\|_{l+2}^{l+2}+\left\|v_{t}\right\|_{l+2}^{l+2}\right)-a_{2}\left(\|\nabla u\|^{2}+\|\nabla v\|^{2}\right)-2 \tau(t) e^{-2 \tau_{1}} \int_{0}^{1}\left(\left\|\nabla z_{1}(x, \rho, t)\right\|^{2}+\left\|\nabla z_{2}(x, \rho, t)\right\|^{2}\right) \mathrm{d} \rho \\
& +a_{1}\left(\left\|\nabla z_{1}(x, 1, t)\right\|^{2}+\left\|\nabla z_{2}(x, 1, t)\right\|^{2}\right)-a_{4}\left(\left\|\nabla u_{t}\right\|^{2}+\left\|\nabla v_{t}\right\|^{2}\right)+a_{5}\left[\left(h_{1}^{\prime} o \nabla u\right)(t)+\left(h_{2}^{\prime} o \nabla v\right)(t)\right] \\
\leq & -m E(t)-c E^{\prime}(t)
\end{aligned}
$$

where $m=\min \left\{\left(2 e^{-2 \tau_{1}} / \xi\right), 2\left(a_{2} / a\right), a_{3}\right\} \quad$ and $\quad\left(a_{1} / \beta\right) \leq c \leq$ $\min \left\{\left(a_{4} / \lambda\right),-2 a_{5}\right\}$.

Let $L(t)=F(t)+c E(t) \sim E(t)$. From (101), we get

$$
L^{\prime}(t) \leq-c^{\prime} L(t), \quad \forall t \geq t_{0},
$$

for some $c^{\prime}>0$. A simple integration over $\left(t_{0}, t\right)$ yields

$$
L(t) \leq L\left(t_{0}\right) e^{-c^{\prime}\left(t-t_{0}\right)}, \quad \forall t \geq t_{0} .
$$

Thanks to equivalence between $L$ and $E$, we obtain (16).

\section{Data Availability}

No data were used to support this study. 


\section{Conflicts of Interest}

The authors declare that there are no conflicts of interest regarding the publication of this paper.

\section{Authors' Contributions}

All authors contributed equally to this study. All authors read and approved the final manuscript.

\section{References}

[1] N. Mezouar and S. Boulaaras, "Global existence and decay of solutions for a class of viscoelastic Kirchhoff equation," Bulletin of the Malaysian Mathematical Sciences Society, vol. 43, no. 1, pp. 725-755, 2020.

[2] N. Mezouar and S. Boulaaras, "Global existence of solutions to a viscoelastic non-degenerate Kirchhoff equation," Applicable Analysis, 2018, in Press.

[3] S. Nicaise and C. Pignotti, "Interior feedback stabilization of wave equations with time dependent delay," Electronic Journal of Differential Equations, vol. 41, pp. 1-20, 2011.

[4] Q. C. Zhong, Robust Control of Time-Delay Systems, Springer, London, UK, 2006.

[5] E. Fridman, S. Nicaise, and J. Valein, "Stabilization of second order evolution equations with unbounded feedback with time-dependent delay," SIAM Journal on Control and Optimization, vol. 48, no. 8, pp. 5028-5052, 2010.

[6] S.-T. Wu, "Asymptotic behavior for a viscoelastic wave equation with a delay term," Taiwanese Journal of Mathematics, vol. 17, no. 3, pp. 765-784, 2013.

[7] K. Daewook, "Asymptiotic behavior for the viscoelastic Kirchhoff type equation with an internal time varying delay term," East Asian Mathematical Journal, vol. 32, no. 3, pp. 399-412, 2016.

[8] S. Nicaise and C. Pignotti, "Stability and instability results of the wave equation with a delay term in the boundary or internal feedbacks," SIAM Journal on Control and Optimization, vol. 45, no. 5, pp. 1561-1585, 2006.

[9] J. Y. Park and J. R. Kang, "Global existence and uniform decay for a nonlinear viscoelastic equation with damping," Acta Applicandae Mathematicae, vol. 110, no. 3, pp. 1393-1406, 2010.

[10] J. L. Lions, Quelques Methodes de Resolution des Problemes Aux Limites Non Lineaires, Dunod, Paris, France, in French, 1969. 